\title{
Design and Ground Calibration of the Helioseismic and Magnetic Imager (HMI) Instrument on the Solar Dynamics Observatory (SDO)
}

\author{
J. Schou • P.H. Scherrer • R.I. Bush • R. Wachter • S. Couvidat • M.C. Rabello-Soares • \\ R.S. Bogart · J.T. Hoeksema · Y. Liu · T.L. Duvall Jr. • D.J. Akin • B.A. Allard • \\ J.W. Miles · R. Rairden · R.A. Shine - T.D. Tarbell • A.M. Title · C.J. Wolfson • \\ D.F. Elmore • A.A. Norton • S. Tomczyk
}

Received: 7 June 2011 / Accepted: 9 August 2011 / Published online: 4 October 2011

(C) The Author(s) 2011. This article is published with open access at Springerlink.com

\begin{abstract}
The Helioseismic and Magnetic Imager (HMI) investigation (Solar Phys. doi:10. 1007/s11207-011-9834-2, 2011) will study the solar interior using helioseismic techniques as well as the magnetic field near the solar surface. The HMI instrument is part of the Solar Dynamics Observatory (SDO) that was launched on 11 February 2010. The instrument is designed to measure the Doppler shift, intensity, and vector magnetic field at the solar pho-
\end{abstract}

The Solar Dynamics Observatory

Guest Editors: W. Dean Pesnell, Phillip C. Chamberlin, and Barbara J. Thompson

Electronic supplementary material The online version of this article (doi:10.1007/s11207-011-9842-2) contains supplementary material.

J. Schou $(\varangle) \cdot$ P.H. Scherrer · R.I. Bush · R. Wachter · S. Couvidat · M.C. Rabello-Soares ·

R.S. Bogart · J.T. Hoeksema · Y. Liu

W.W. Hansen Experimental Physics Laboratory, Stanford University, Stanford, CA 94305-4085, USA

e-mail: schou@sun.stanford.edu

T.L. Duvall Jr.

Laboratory for Astronomy and Solar Physics, NASA Goddard Space Flight Center, Greenbelt, MD 20771, USA

D.J. Akin - B.A. Allard · J.W. Miles · R. Rairden · R.A. Shine · T.D. Tarbell · A.M. Title · C.J. Wolfson Lockheed Martin Solar and Astrophysics Laboratory, 3251 Hanover St., Palo Alto, CA 94304, USA

J.W. Miles

USRA NASA Ames Research Center, Mail Stop 211-3, Moffett Field, CA 94035, USA

D.F. Elmore · A.A. Norton · S. Tomczyk

High Altitude Observatory, National Center for Atmospheric Research, 3080 Center Green CG-1, Boulder, CO 80301, USA

Present address:

D.F. Elmore

National Solar Observatory/Sacramento Peak, 3010 Coronal Loop, Sunspot, NM 88349, USA

Present address:

A.A. Norton

James Cook University, School of Engineering and Physical Sciences, Townsville, QLD, 4810,

Australia 
tosphere using the $6173 \AA$ Fe I absorption line. The instrument consists of a front-window filter, a telescope, a set of waveplates for polarimetry, an image-stabilization system, a blocking filter, a five-stage Lyot filter with one tunable element, two wide-field tunable Michelson interferometers, a pair of $4096^{2}$ pixel cameras with independent shutters, and associated electronics. Each camera takes a full-disk image roughly every 3.75 seconds giving an overall cadence of 45 seconds for the Doppler, intensity, and line-of-sight magnetic-field measurements and a slower cadence for the full vector magnetic field. This article describes the design of the HMI instrument and provides an overview of the pre-launch calibration efforts. Overviews of the investigation, details of the calibrations, data handling, and the science analysis are provided in accompanying articles.

Keywords Solar Dynamics Observatory · Helioseismology, observations · Instrumentation and data management $\cdot$ Magnetic fields, photosphere

\section{Introduction}

The Helioseismic and Magnetic Imager (HMI) instrument was built as part of the HMI investigation (Scherrer et al., 2011) and is designed to measure the Doppler shift, line-ofsight magnetic field, intensity, and vector magnetic field at the solar photosphere using the $6173 \AA$ A Fe I absorption line (Norton et al., 2006).

To a significant extent the HMI instrument design is based on the highly successful Michelson Doppler Imager (MDI) instrument (Scherrer et al., 1995), but it does have a few significant improvements. Among these improvements are two cameras instead of one, better spatial resolution and temporal coverage, the capability to observe the full Stokes vector, full-disk filtergram data that are all downlinked without significant processing, and a significant level of redundancy. The minimal onboard processing ensures that optimal processing can be done, and if necessary redone, on the ground.

Like MDI, the HMI instrument is what has become known as a filtergraph. In other words it takes a series of images at different wavelengths and polarizations that are combined to derive the physical parameters. This is in contrast with spectrographs that take spectra while scanning across the image.

Below we start with a description of the various components of the instrument (Section 2). This is followed by an overview of the calibration in Section 3 and conclusions in Section 4. Rather than describing all the instrument details and calibrations in one article, a number of other articles describe the main components of the calibration efforts. Wachter et al. (2011) describe the image quality and CCD performance, Couvidat et al. (2011) the filter performance, and Schou et al. (2010) the polarization properties. In all cases we have restricted ourselves to ground-based results, with updates to be made and published after analysis of on-orbit HMI data.

The HMI instrument was built by the Lockheed Martin Solar and Astrophysics Laboratory (LMSAL). Many of the components used in HMI are essentially identical to those used for the Atmospheric Imaging Assembly (AIA) instrument also built at LMSAL, for which more details can be found in Lemen et al. (2011).

\section{Instrument Description}

The HMI instrument consists of three main parts: an optics package, an electronics box, and a harness to connect the two. 
The HMI Optics Package (HOP) layout is described in Section 2.1. We present details of the imaging optics in the HOP in Section 2.2, polarization selectors in Section 2.3, filters in Section 2.4, the Image-Stabilization System (ISS) in Section 2.5, the various mechanisms in Section 2.6, the thermal control in Section 2.7, and the CCDs and cameras in Section 2.9. The contents of the HMI Electronics Box are described in Sections 2.8 and 2.10, while the flight software is described in Section 2.11. The harness is not described in detail. Finally, Section 2.12 contains a comparison of MDI and HMI.

For reference, the masses are $47 \mathrm{~kg}$ for the optics package, including Multi-Layer Insulation (MLI), $19 \mathrm{~kg}$ for the electronics package, and $7 \mathrm{~kg}$ for the harness, for a total of $73 \mathrm{~kg}$. The HMI instrument uses about $95 \mathrm{~W}$ of power during normal operations, of which about $15 \mathrm{~W}$ are consumed by each camera.

\subsection{HMI Optics Package}

The HOP houses the optical components of the instrument and protects them from the environment. The bottom of the HOP is an optical bench consisting of two face sheets and a honeycomb core. The side panels and a number of internal partitioning walls are mounted on this bench and in turn support the lid of the box. The box is packaged in MLI to provide thermal stability and to minimize the amount of power needed to keep the HOP near the design temperature of around $20 \mathrm{C}$. A number of heaters are attached to the bottom and top of the box. Their placement and operation is described in Section 2.7.1. The combination of the insulation system and the multiple heaters means that it is possible to keep the HOP at an adjustable and stable temperature.

Two CCDs and attached radiators are mounted on top of the box along with two Camera Electronics Boxes (CEBs), also with radiators. The camera components are described in Section 2.9.

The HOP mounts to the spacecraft using four fiberglass legs, two of which are also used to point the HOP, as described in Section 2.6.4.

Finally, a connector panel on the side of the box (near the shutters) connects to the harness. Vents ensure that the pressure differential between the inside and outside of the HOP stays low during launch and provide an escape path for any volatile contaminants in the box.

To provide an overview, a schematic of the optical path of HMI is shown in Figure 1 and a photograph of the inside of the HOP in Figure 2. Sunlight travels through the instrument from the front window at the upper right to the cameras at the lower right of the schematic. The majority of the optical components are mounted to the optical bench. The components along the optical path are described in more detail in the following subsections. A raytrace is shown in Figure 3. For a prescription of the optical components see Appendix B.1. In order to avoid radiation-induced darkening, all glass elements used are made of radiation-resistant glass, except for one element in the front window and parts of the Michelsons.

The front window is a $50 \AA$ bandpass filter that reflects most of the incident sunlight. It is followed by a $14-\mathrm{cm}$ diameter refracting telescope consisting of a primary and secondary lens.

Two focus/calibration mechanisms, three polarization-selection mechanisms, and the ISS tip-tilt mirror are located between the telescope and the polarizing beamsplitter feeding the tunable filter. The filter is mounted in a precisely temperature-controlled oven, containing the following elements:

- A telecentric lens

- A blocking filter

- A Lyot filter with a single tunable element 


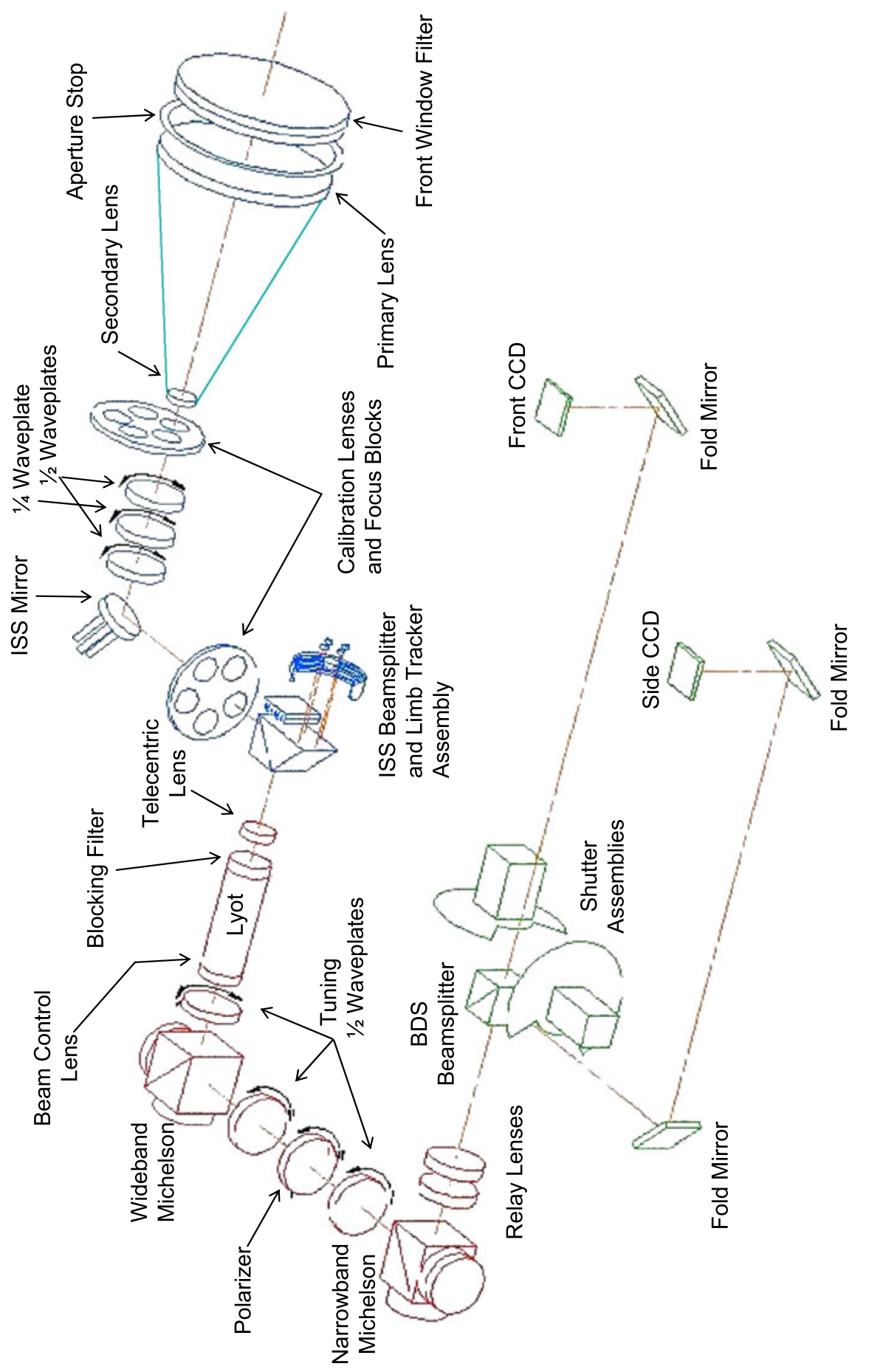


Figure 2 Photograph of the inside of the optics package. Light enters through the telescope at the upper left, passes through the chain of filters near the bottom, and is detected by the CCD cameras at the upper right. The box is roughly $88 \mathrm{~cm}$ long by $55 \mathrm{~cm}$ wide by $16 \mathrm{~cm}$ high. The various elements are identified in Figure 1.

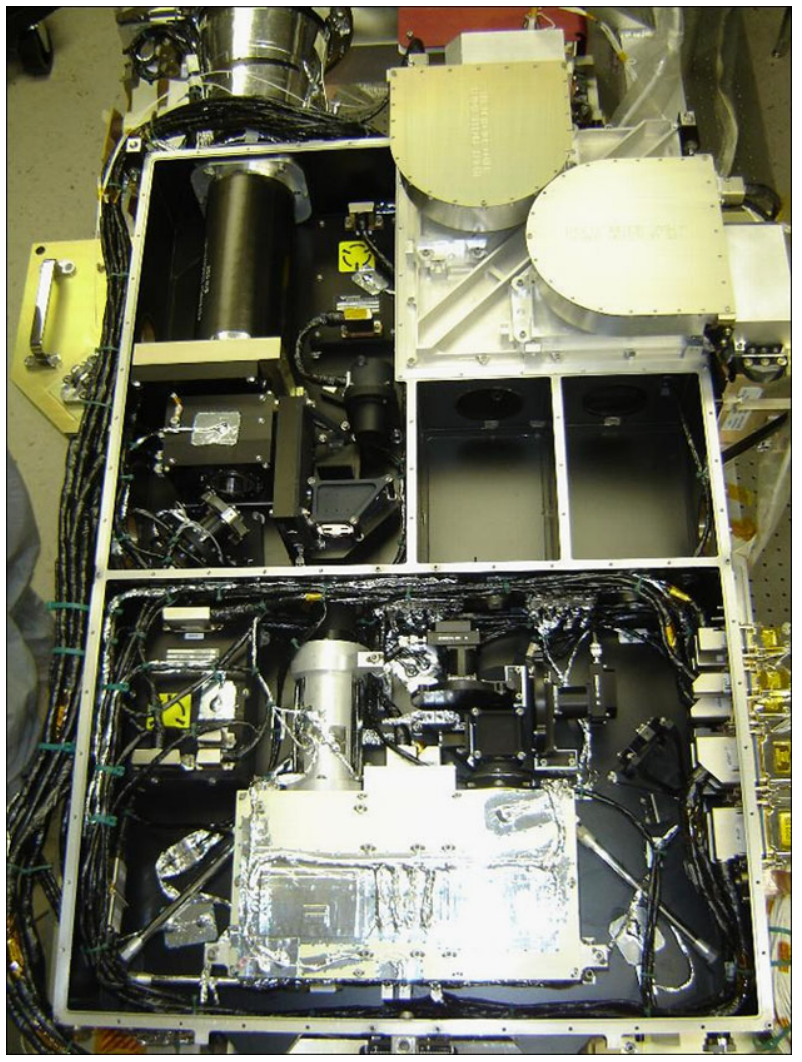

- A beam control lens

- Two tunable Michelson interferometers

- Reimaging optics

Following the oven is the Beam Distribution System (BDS) beam splitter that feeds two identical shutters each mounted at a pupil image, and the CCD camera assemblies at the focal plane.

In a number of places $1 / 4$ waveplates are included to reject unwanted reflections. These are not always shown or discussed further, but are included in the Zemax ${ }^{\circledR}$ prescription in Appendix B.1. In total the light traverses 80 or 84 elements, depending on the path though the Michelsons (and counting multiple traverses of the same element) and is reflected seven or nine times.

There are two mechanisms external to the optics package: a front door, which protects the front window during launch, and an alignment mechanism used to adjust the optics-package pointing.

The following subsections describe each subsystem in more detail.

\subsection{Imaging Optics}

The main telescope is a two-element refracting telescope with a $140 \mathrm{~mm}$ clear aperture, giving an image with a nominal diffraction limit $(\lambda / D)$ of 0.91 arcsec. The primary lens is convex aspherical on the front surface and convex spherical on the back surface, while 
Table 1 Key numbers for the imaging optics. EFL is the Effective Focal Length. Image Size is the diameter of the solar image for a solar radius of $960 \mathrm{arcsec}$, corresponding, roughly, to the mean over the year. Numbers for the primary lens only include the front window and primary lens. Numbers for the telescope include only the front window, primary, and secondary lenses. Path lengths are from the front surface of the front window.

\begin{tabular}{lllllr}
\hline Subsystem & EFL & Focal Ratio & Image Size & Angular Magn. & Path Length \\
\hline Primary Lens & $586 \mathrm{~mm}$ & $\mathrm{f} / 4.18$ & $5.45 \mathrm{~mm}$ & 1.00 & $615 \mathrm{~mm}$ \\
Telescope & $2468 \mathrm{~mm}$ & $\mathrm{f} / 17.65$ & $23.0 \mathrm{~mm}$ & 5.43 & $895 \mathrm{~mm}$ \\
Complete & $4953 \mathrm{~mm}$ & $\mathrm{f} / 35.42$ & $46.1 \mathrm{~mm}$ & 8.24 & $2218 \mathrm{~mm}$ \\
\hline
\end{tabular}

the secondary (Barlow) lens is bi-concave. The primary and secondary lenses are connected with a low coefficient-of-thermal-expansion metering tube made of a carbon fiber composite to maintain focus. Key parameters of the optical system are given in Table 1. All surfaces other than the front of the primary are spherical.

The focal ratio (in vacuum) through the Lyot is f/18.3 and through the Michelsons f/21.7. The primary to secondary magnification is thus 2.01 of which a factor of 1.63 is accomplished by the reimaging lenses.

The telephoto ratios of the telescope and the entire system are 2.72 and 2.23 , respectively, measuring from the front face of the front filter.

The HMI calibration and focus-adjustment system consists of two calibration-focus wheels (see Section 2.6.2), each of which contain an empty position, three positions with optical flats of varying thickness, and a lens in the fifth position. By making the (identical) steps in optical thickness in the one wheel four times larger than the steps in the other wheel, it is possible to select one of 16 uniformly spaced focus steps of roughly $1 \mathrm{~mm}$ at the focal plane, corresponding to $2 / 3$ of a depth of focus. Note that the heaters on the front-window mounting ring (see Section 2.7.1) can also be used to fine tune the focus by inducing a center-to-edge temperature gradient in the front window, thereby causing it to act as a weak lens. Besides allowing best focus to be set on orbit, this capability also provides a highly repeatable means for measuring the instrument focus and assessing image quality through phase-diversity analysis.

In calibration mode, a lens in the fifth position of each wheel images the entrance pupil onto the focal plane to provide uniformly integrated sunlight. This is illustrated in Figure 4. Calibration-mode images are used to provide Doppler calibrations, monitor the instrument transmission and assess variations in the flatfield. The calibration-mode image has a diameter of $50.1 \mathrm{~mm}$, large enough to cover the largest solar image seen.

The light is folded by the ISS mirror and then split by a polarizing beamsplitter to send the s-component light to the filters while passing the orthogonal light onto the limb sensor. The limb sensors receive the full $50 \AA$ bandwidth of the front window, while light for the rest of the instrument continues through an $8 \AA$ blocking filter located just inside the oven.

A telecentric lens at the entrance of the filter oven produces a telecentric beam for the Lyot filter. This ensures that the angular distribution of light passing through the Lyot filter is identical for each image point, minimizing the variation of the central wavelength across the image as well as contrast loss. However, different parts of the solar image pass through different parts of the filters.

At the exit of the Lyot filter a beam-control lens minimizes the clear-aperture requirements for the Michelsons by making the extreme rays parallel. This means that the beam is not telecentric through the Michelson interferometers.

Doing so causes a center-to-edge variation of the center wavelength and a degradation of the contrast. However, this degradation, as well as the consequent increase in the noise, 
Figure 3 Raytrace of HMI, excluding the path to the limb sensor. The CCD detectors are above the plane of the plot. Different colors indicate different places on the solar image, at center and limb on each side. See Figure 1 for identification of the elements. The vertical distance from the front of the front window to the back of the second Michelson is $106 \mathrm{~cm}$.

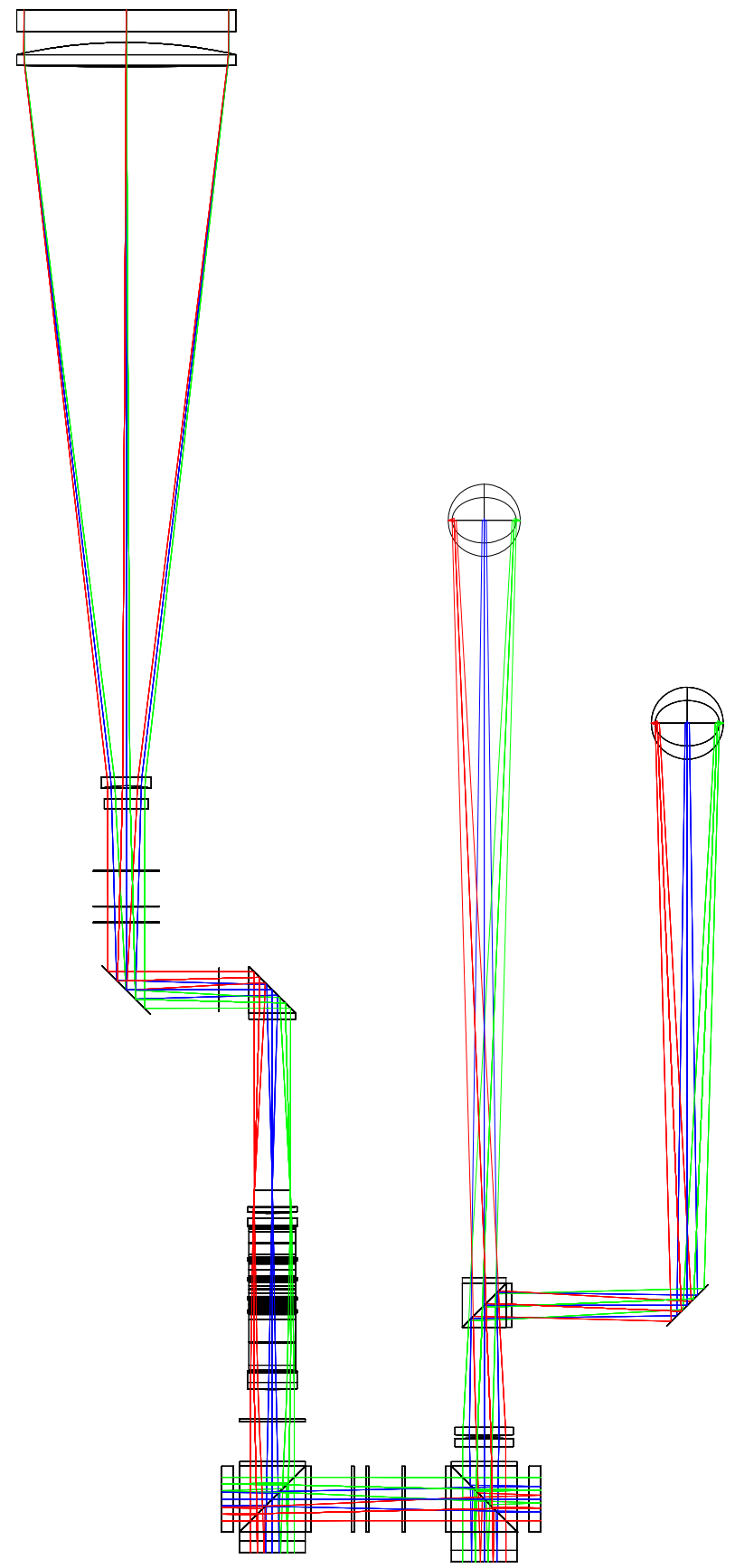

is insignificant both in an absolute sense and relative to that introduced by imperfections in the manufacture of the Michelsons. As the actual center-wavelength variation and contrasts are measured (see Section 3.3), the various causes are largely of academic interest. 


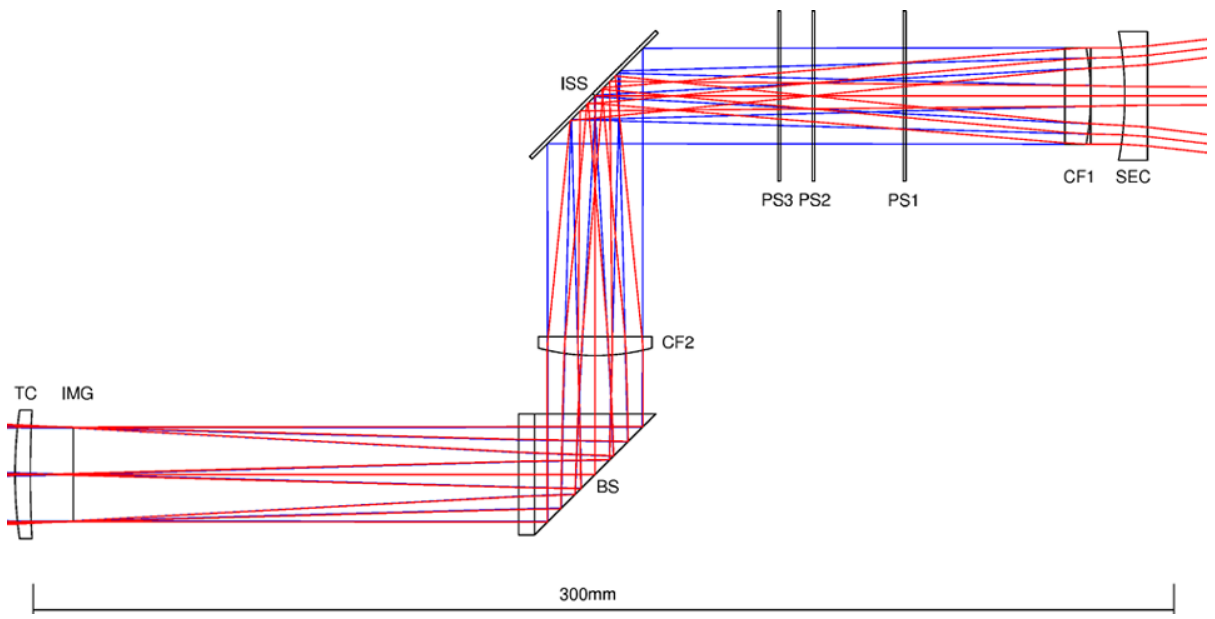

Figure 4 Raytrace with obsmode rays shown in blue and calmode rays shown in red (see Section 2.2). In the order the light travels (from upper right to lower left) the elements shown are: Secondary lens (SEC), first calibration lens (CF1), three polarization selectors (PS1, PS2, and PS3), ISS fold mirror (ISS), second calibration lens (CF2), polarizing beamsplitter (BS), a line showing the primary focus (IMG), and telecentric lens (TC) (Schou et al., 2010).

At the exit of the oven, a pair of lenses reimages the primary focus onto the detectors. A beamsplitter evenly divides the light between the two camera paths with folding mirrors used to provide convenient placement of the cameras on top of the optics package. In order to prevent non-uniform movement of the shutter blades causing exposure gradients across the image, the shutters are placed at a pupil image. This has the minor disadvantage of briefly apodizing the aperture causing a slight broadening of the PSF. (Note that the shutter blade takes roughly $10 \mathrm{~ms}$ to cross the beam, which is small, but not negligible, compared to a nominal exposure time of around $150 \mathrm{~ms}$.) The alternative of using a focal-plane shutter was rejected as it is likely to cause uncorrectable spatial non-uniformities in the exposures.

The unused port of the beamsplitter has a light trap. For the purpose of having a light source to test the cameras when there is no external light source, an LED was placed at this port. There is no need to use this LED on orbit after the front door has been opened.

\subsection{Polarization Selectors}

The polarization selectors rotate quartz waveplates to convert the desired incoming polarization into the fixed linear polarization selected by the polarizing beamsplitter. Each retarder is mounted in a Hollow Core Motor (HCM) that allows it to be rotated to any of 240 uniformly spaced angles (see Section 2.6.1). The nominal retardances of the waveplates are 10.5, 10.25, and 10.5 waves, in the order encountered. This design has a three for two redundancy, meaning that any one of the three waveplates can be left in any position, while preserving the ability to measure all polarization states (subject to the finite number of positions). High-order waveplates were chosen for mechanical convenience, but this choice does have implications for the polarization performance, as discussed by Schou et al. (2010). In particular, it leads to artifacts in calmode where the angles of incidence are substantially larger than in observation mode (obsmode), and thus means that the calibration mode (calmode) results cannot be applied to obsmode without corrections. 


\subsection{Filters}

The HMI filter system consists of the front window, a blocker filter, a Lyot filter with a single tunable element, and two tunable Michelson interferometers. Both the Lyot filter and the Michelsons have temperature-compensating designs, and all of the filters, except the front window, are mounted in an oven stable to better than $\pm 0.01 \mathrm{C} \mathrm{hour}^{-1}$, as discussed in Section 2.7.2. The filter system enables narrow-band filtergrams to be made across the Fe I $6173 \AA$ line by co-tuning one Lyot tunable element and both Michelson interferometers. The combined filter bandpass is $76 \mathrm{~m} \AA$ FWHM (nominally at center tuning) with a tunable range of $690 \mathrm{~m} \AA$. Details of the filters are given by Couvidat et al. (2011) and summarized below in the order encountered by the light.

\subsubsection{Front Window}

The front window, the main purpose of which is to limit the heat input to the instrument, is a $50 \AA$ bandpass filter made by Andover Corporation. In the order in which the light travels it consists of $6 \mathrm{~mm}$ of Schott BK7G18 radiation-resistant clear glass, $3 \mathrm{~mm}$ of Schott GG495 green glass, a bandpass coating, an infrared rejection coating, and another $6 \mathrm{~mm}$ of BK7G18 glass. The BK7G18 glass, among other things, serves as a radiation shield for the non-radiation-resistant GG495 glass. The GG495 glass serves two important purposes: One is to block the blue and ultraviolet light from reaching the bandpass filter, which can be damaged by exposure to those wavelengths. Another is to cause the window to absorb a small amount of light, thereby heating it up and limiting the center-to-edge temperature gradient.

A particular concern for the front window is that it experiences a very large temperature perturbation (tens of $\mathrm{C}$ ) when going in and out of eclipses, causing the focus to change dramatically due to the induced center-to-edge temperature gradient (and to some extent due to the temperature change of the primary lens, which is radiatively coupled to the window). The eclipses are up to 72 minutes in length and so in order to limit the additional loss of data while recovering, a limit on the recovery time of 60 minutes was imposed. Models of the front window indicate an e-folding time of order ten to fifteen minutes for any temperature perturbations, which should be adequate to meet the requirement. Models indicate that raising the temperature of the window mounting ring (using the operation heaters described in Section 2.7.1) during the eclipses should help with the recovery.

Despite the use of radiation-resistant glass, a certain increase in the absorptivity, as seen with MDI, is likely to occur, which may in turn change the focus of the instrument. This focus change may be compensated by changing the focus position being used and/or fine tuned by changing the front-window heater setpoints.

Due to manufacturing problems there was a significant wavefront error in all of the front windows produced and as a result a compensating error was polished into the surface of the selected front window. This is further discussed by Wachter et al. (2011).

Some of the optical elements, including the front window, cause fringes in obsmode and/or calmode, as discussed by Couvidat et al. (2011).

\subsubsection{Blocking Filter}

The blocking filter is a three-period, all-dielectric, interference filter, also made by Andover Corporation, with a FWHM bandpass of $8 \AA$. The blocker rejects the unwanted orders of the Lyot and Michelson filters and limits the heat input into the oven. 
Figure 5 A pair of Michelson interferometers. The protective yellow tape on the vacuum legs was removed before launch. The cubes are $45 \mathrm{~mm}$ on each side. Photo courtesy of LightMachinery Inc.

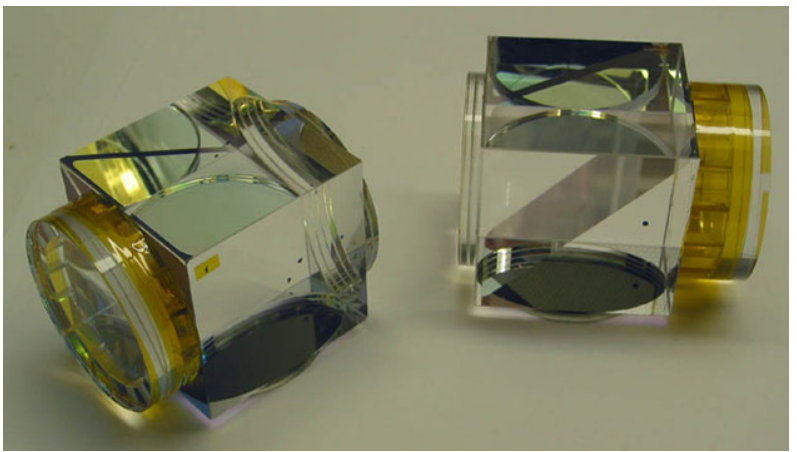

\subsubsection{Lyot Filter}

The wide-field, temperature-compensated Lyot filter uses the same basic design as the MDI (Scherrer et al., 1995) filter with the addition of a fifth tuned element in order to accommodate the wide range in radial velocity due to the geosynchronous orbit of SDO. The fiveelement (named E1 through E5 in order of increasing FWHM) Lyot filter has a 1:2:4:8:16 design (actual order is given in Section 2.4.5). This is unlike the MDI filter where some of the elements were doubled in order to reduce unwanted sidelobes in the untuned part. The final (narrowest) filter element of the Lyot filter is tuned by a rotating half-wave plate mounted in a HCM identical to those used for the polarization selectors. The Lyot tuning waveplate is not redundant. The FWHM of the untuned part is $612 \mathrm{m \AA}$, nominally. The Lyot components are held in optical contact by optical grease to avoid stressing the elements with changing temperature, and are keyed to hold the elements in proper relative alignment.

Each Lyot element contains nine pieces: A fused-silica spacer, a polarizer, a piece of potassium dihydrogen phosphate (KDP) or ammonium dihydrogen phosphate (ADP), a piece of calcite, a half waveplate, a piece of calcite, a piece of KDP or ADP, a quarter waveplate, and a fused-silica spacer. In addition the first element (E2) has a quarter waveplate between the first spacer and polarizer. By pairing KDP (for E1, E2, and E3) or ADP (for E4 and E5) elements with the calcite elements, the temperature sensitivity in the calcite is compensated by an opposite change in the KDP or ADP.

Details of the construction of the individual elements can be found in the supplementary material described in Appendix B.1. Physical diameters of the Calcite, ADP, and KDP elements are $32 \mathrm{~mm}$ with a clear aperture of $30 \mathrm{~mm}$.

Due to a drawing error, the clear aperture at the exit of the Lyot holder is only marginally larger than the solar image. This results in some vignetting if the image is not accurately centered with respect to the obstruction. The position of the obstruction is expected to be very stable and can be determined from the regular flatfield observations taken every few months. For details see Wachter et al. (2011).

\subsubsection{Michelson Interferometers}

The final filters are two wide-field, tunable, solid Michelson interferometers with clear apertures of $32 \mathrm{~mm}$ and nominal free spectral ranges of $345 \mathrm{~m} \AA$ and $172 \mathrm{~m} \AA$ (172 $\mathrm{m} \AA$ and $86 \mathrm{~m} \AA$ FWHM pass bands, respectively, both made by LightMachinery). The design incorporates a polarizing beamsplitter with a vacuum leg and a solid glass leg. The vacuum leg is maintained with temperature-compensating standoffs made of calcium fluoride. The Michelsons are pictured in Figure 5. 
Figure 6 Example of HMI tuning-position profiles obtained from the wavelength-dependence calibration procedure. Six tuning positions are shown here with respect to the Fe I solar line at disk center and at rest. The line profile was provided by R.K. Ulrich and obtained at the Mount Wilson Observatory. Details of the calibration from which the profiles are derived are given in Section 3.3.

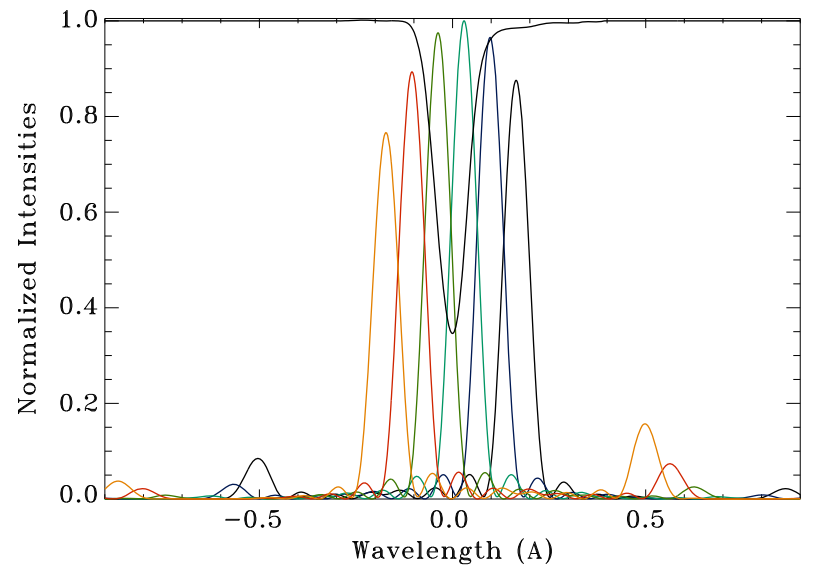

Tuning of the two Michelsons is accomplished by rotating a combination of a half-wave plate, a polarizer, and a half-wave plate, each of which is mounted in a HCM identical to those used for the polarization selectors. Unlike the Lyot tuner, this design has a full threefor-two redundancy, meaning that any one of the three mechanisms can fail in any position without impacting the ability to tune either Michelson. Ordinarily only the waveplates are used for tuning.

In order to ensure the best spatial uniformity of the tuning, regular non-radiation-resistant BK7 glass was used for the Michelson cubes. Given the substantial amount of glass between the edge of the beam and the amount of additional shielding around the Michelsons, no significant amount of radiation-induced darkening is expected.

\subsubsection{Filter Summary}

Figure 6 shows a model of the transmission profiles of the resulting filter. Details of the actual performance are given by Couvidat et al. (2011).

As with the MDI Michelsons, some amount of wavelength drift may be expected with time. Any such drift can be compensated by changing the HCM positions corresponding to each desired tuning position.

In summary the nominal filter FWHMs are, in the order in which the light travels:

- Front window: $50 \AA$

- Blocking filter: $8 \AA$

- Lyot element E2: $0.69 \AA$

- Lyot element E3: $1.38 \AA$

- Lyot element E4: $2.76 \AA$

- Lyot element E5: $5.52 \AA$

- Lyot element E1: 344 mÅ (tuned)

- Wide Band (WB) Michelson: $172 \mathrm{~m} \AA$ (tuned)

- Narrow Band (NB) Michelson: 86 mÅ (tuned)

- Untuned part: $612 \mathrm{m \AA}$

- Complete filter: $76 \mathrm{~m} \AA$ (at center tuning position)

Using the HCMs with half waveplates for tuning and having 240 steps means that one step is $1 / 60$ of the Free Spectral Range (FSR) or 1/30 of the Full Width at Half Maximum (FWHM) of the corresponding tuned element. 


\subsection{Image-Stabilization System}

The HMI Image-Stabilization System is a closed-loop system with a tip-tilt mirror to remove jitter measured at a primary image within HMI.

The ISS uses the light rejected by the polarization-selection beamsplitter to image the Sun at the guiding-image focal plane, located at the opposite side of the ISS beamsplitter from the Lyot filter (see Figure 1). Four detectors are positioned on the north, south, east, and west limbs of the solar image, each consisting of a redundant photodiode pair. The electronic limb-sensor photodiode preamplifier has two gains, ground-test mode and Sun mode, and selectable prime or redundant photodiodes. In order to avoid the introduction of noise between the limb sensors and the electronics package the (redundant) limb tracker pre-amp board is mounted inside the optics package.

The mirror uses low-voltage piezoelectric transducer (PZT) actuators to remove errors in the observed limb position, derived as the difference between the intensity reaching opposite photo diodes. The mirror design has a first resonance of $\approx 800 \mathrm{~Hz}$, which is much higher than the structural modes of the HMI optics package and the expected jitter frequencies. This enables a simple analog control system.

The range of the tilt mirror is approximately \pm 18 by \pm 15 arcsecs in image space. A larger range for coarse pointing is provided by adjustable instrument legs described in Section 2.6.4.

The servo gains and other parameters are adjustable by ground commands. In particular, offsets can be added to the $X$ - and $Y$-axis error signals to change the nominal pointing while maintaining lock. Individual PZT actuator offsets can be specified to set the nominal position of the mirror anywhere in its range during open-loop operation or during calibrations.

The error and mirror signals are continually sampled, and downlinked at a cadence of four to eight seconds to monitor jitter and drift. For calibration purposes, these signals can be downlinked at a rate of up to $512 \mathrm{~Hz}$ allowing for a more detailed measurement of the performance.

\subsection{Mechanisms}

The instrument contains a number of different types of mechanisms, each of which is described below. In most cases the details of their operation are of little consequence to the scientific operation of the instrument, in other cases they have important implications. The mechanisms are controlled by Field Programmable Gate Arrays (FPGAs) commanded by the flight software.

\subsubsection{Hollow Core Motors}

The HCMs are used to rotate the polarization selectors and wavelength tuning optics. Each motor has an encoder with 240 positions. To move from one position to another the motor is first commanded to move in the desired direction. Once the encoder indicates that a particular position has been reached, the FPGA waits a programmable time after which the motor windings are shorted out, causing the motor to brake and stop some distance away. By selecting an appropriate encoder position and delay time (selectable in units of $1 \mu \mathrm{s}$ ), the motors can be made to stop at any desired position (there is very little detent in the motors). However, in order to be able to control the stopping positions more accurately, only positions at the places where the encoder changes are used. This is further discussed in Section 2.11.5. A HCM is shown in Figure 7. 
Figure 7 A Hollow Core Motor. The size of the housing is $88.9 \mathrm{~mm}$ by $101.6 \mathrm{~mm}$ by $24.0 \mathrm{~mm}$.

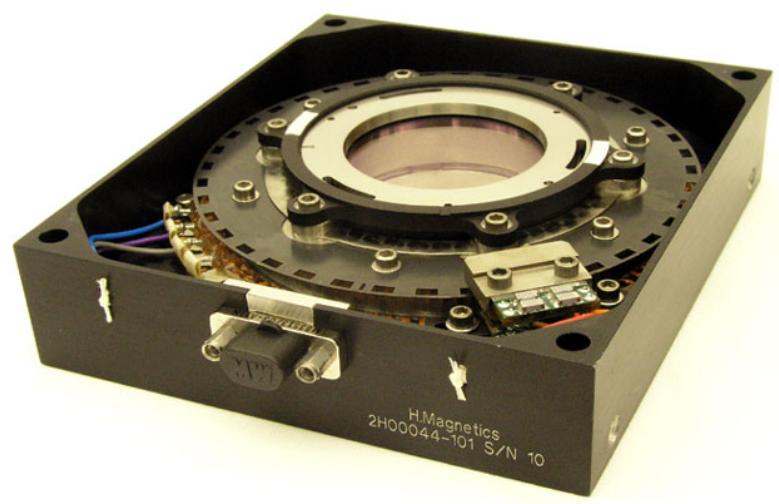

The $360^{\circ}$ spin time of a HCM is around one second. By going in the shortest direction it is thus possible to change between arbitrary positions in less than about $500 \mathrm{~ms}$.

Since the HCMs are planned to move as often as once every 1.875 seconds and thus will experience about 80 million moves in five years, an extensive life-test program was performed in which 375 million moves were performed without problems, leading to a high confidence that they will perform as desired.

The standard deviation of the stopping positions depends significantly on the motor being tested and the starting and stopping positions, but is generally of order tens of arcsecs of rotation of the motors.

When the adjustment algorithm discussed in Section 2.11.5 is used, the accuracy of the absolute stopping positions is set by the accuracy of the encoder, which is specified to be $0.15^{\circ}(0.1$ steps $)$ or better. Separate measurements with a precision encoder show that the accuracy is indeed better than this.

\subsubsection{Focus Wheels}

The focus wheels are very similar to the HCMs, except that the optics are placed around the rotation axis in a wheel, rather than on axis. While they can, in principle, be positioned at any one of 180 positions, only the five positions for which the optics are centered are used. The focus wheels also take about one second to rotate, and since the longest distance necessary to move is 0.4 revolutions, the maximum move time is $400 \mathrm{~ms}$. A photograph of a filterwheel is shown in Figure 8.

As relatively few moves are needed and since similar mechanisms have been flown on LMSAL instruments before, no formal life test was performed.

\subsubsection{Shutters}

The shutters are very similar to those on MDI and the Solar X-ray Imager for GOES (Lemen et al., 2004) and provide exposure measurements with a resolution of $1 \mu$ s. The combination of knowing the actual exposure times and downlinking all of the images means that it is possible to correct for any exposure variations. Thus even variations more than an order of magnitude larger than those seen on MDI after about 150 million operations (currently the errors are of order a part in 10000 of the exposure time) on orbit will cause no detrimental effects. A shutter is shown in Figure 9. 
Figure 8 Focus wheel. In this case the one closest to the telescope. Clockwise from the top are the thinnest focus block, the thickest focus block, the blank position, the medium focus block, and the calibration lens. The size of the housing is $109.98 \mathrm{~mm}$ by $121.03 \mathrm{~mm}$ by $19.05 \mathrm{~mm}$.

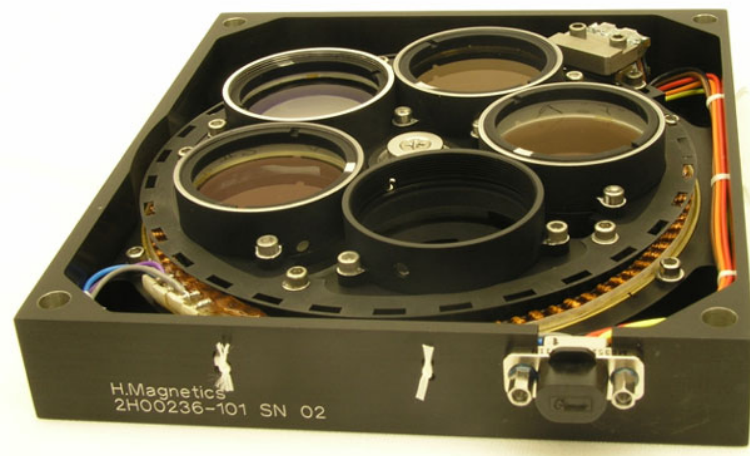

Figure 9 Shutter mechanism and blade. For scale, the shutter blade has a diameter of $84.07 \mathrm{~mm}$.

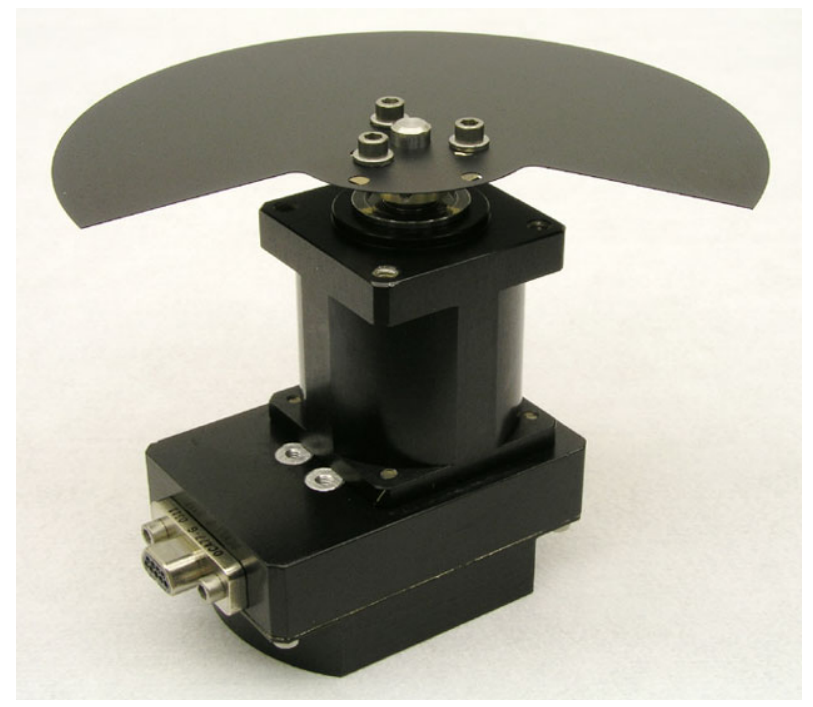

In normal operation, the shutters can be commanded to expose the CCDs from about $35 \mathrm{~ms}$ to about $16.7 \mathrm{~s}$ in increments of $1 \mathrm{~ms}$. The expected exposure time on orbit is around $150 \mathrm{~ms}$.

Since the shutters will move roughly once per 3.75 seconds, 40 million exposures are expected in five years. A life test to 85 million exposures was performed, again without problems. At the end of the life test, the typical standard deviation in the exposure time was roughly $10 \mu \mathrm{s}$, meeting the required performance.

\subsubsection{Alignment Mechanism}

The HMI alignment mechanism has two legs, each of which can adjust the optics package pointing in steps of 0.32 arcsec at a rate of 16 steps per second. The directions of image motion caused by the two legs are roughly orthogonal and correspond roughly to diagonal motions in the CCD image. The legs do not have built-in encoders, rather they rely on a hardware center indicator and a software step counter. There are also end-of-range indicators, which if tripped will cause the FPGA to stop moving the leg. These indicators are at positions in the range 1667 to 1850 steps from the center position. The flight software 
also limits the motion to \pm 1500 steps or about \pm 480 arcsecs in order to not rely on the end-of-range indicator.

The alignment legs will have to adjust the pointing by a few steps, perhaps every couple of weeks, to keep the solar image centered in the ISS dynamic range. Otherwise they are not planned to be used after launch. Given this, no formal life test was performed.

\subsubsection{Front Door}

The front door has two identical actuators, either of which can open the door regardless of the position of the other, thus providing redundancy. To close the door, both mechanisms must be in the closed position, but since it is only planned to operate (open) the door once after launch (the purpose of the door is to protect against damage and contamination during integration, test, and launch), there is no need for this to be redundant. The door mechanism has switches that indicate when either of the actuators is in the closed position, and switches that indicate when the door is fully opened (with either actuator).

\subsection{Thermal Control}

\subsubsection{Heaters}

Three different types of heaters are used on the instrument, each of which is redundant.

The survival heaters keep the instrument warm enough to avoid damage when the instrument is not powered, or in case of instrument failure. These heaters are powered directly by the spacecraft (bypassing the instrument electronics) and are controlled by separate built-in thermostats.

Operational heaters are used to maintain the instrument at the desired operating temperature. There are seven such heaters on the instrument: Two on the bottom of the HOP, two on top, one on the telescope tube, and two on the window mounting ring. Each of these zones is controlled by flight software using temperature measurements from thermistors distributed around the optics package, as further discussed in Section 2.11.4.

The placement of the various thermistors and the operational and survival heaters is included as supplementary material. See Appendix B.2.

The CCDs have their own radiators and are ordinarily kept below $-50 \mathrm{C}$ (without any thermal control) to minimize dark current and radiation damage. Decontamination heaters are used during the commissioning phase to warm up the CCDs in order to prevent them from accumulating condensable materials outgassing from the rest of the instrument after launch. These are mounted on the back of the CCDs and are thermostatically controlled. They can be individually enabled and disabled by command. If needed, they can also be used later in the mission to drive off any condensed material or to anneal the CCDs.

\subsubsection{Filter Oven}

To further stabilize the performance of the most temperature-sensitive elements of the filter system, i.e. the Lyot filter and Michelsons, are placed in a filter oven.

The oven can be set to eight uniformly spaced temperatures between $28 \mathrm{C}$ and $35 \mathrm{C}$ and is designed to maintain a temperature stable to $0.01 \mathrm{Chour}^{-1}$, with the best estimate of the actual performance being more than an order of magnitude better. To achieve this performance, the oven uses an analog proportional heater control. The oven-control electronics and heaters are redundant. In order to stabilize the temperature, the oven pre-amp and controller boards are mounted on the oven itself, thereby making them temperature controlled. 
The oven includes a multi-stage thermal isolation system to ensure that the time scale of any residual filter-temperature variations is very long compared to that of the solar $p$-mode oscillations.

The telecentric and relay lenses are the entrance and exit windows of the oven.

\subsection{HMI Electronics Box}

The power supplies, control electronics, and data-processing electronics are housed in a separate HMI Electronics Box (HEB), which is mounted inside the spacecraft bus. The HEB contains a total of 17 items:

- Two Power Converter Subsystems

- Two PCI to Local Bus Bridge and 1553 Interface Boards

- Two RAD6000 Processor Boards

- Four combined Mechanism and Heater Controller Boards (Sections 2.11.4 and 2.11.5)

- One Housekeeping Data Acquisition Board (Section 2.11.3)

- Two Camera Interface (CIF) Boards (Section 2.10.1)

- Two combined Data Compression and High Rate Interface (DCHRI) Boards (Section 2.10.2)

- One ISS Limb Tracker Board (Section 2.5)

- One ISS PZT Driver Board (Section 2.5)

The main control is performed by a redundant pair of BAE Systems RAD6000 CPUs, either of which can control the instrument, as selected when power is applied. The CPU communicates with the spacecraft over a MIL-STD-1553 bus through the Bridge Boards which are also used to connect to the other boards in the HEB.

The power supplies are redundant and are both connected to each of two spacecraft power buses.

The Bridge Boards each contain a very stable temperature-compensated clock, with a finely adjustable frequency, used for time-stamping the images. By monitoring the clockto-spacecraft and spacecraft-to-ground time differences and adjusting the frequency, it is possible to both keep an accurate time (better than $100 \mathrm{~ms}$ ) and to avoid jumps in time, as would be seen if one relied on either the spacecraft time or needed to reset the HMI clock to make adjustments.

As described in Sections 2.5, 2.7.2, and 2.9, the HOP and cameras also contain a number of circuit boards.

A simplified block diagram is shown in Figure 10.

\subsection{CCDs and Camera Electronics}

The CCDs and camera electronics are very similar to those used for AIA (Lemen et al., 2011), except that the HMI CCDs are front-side illuminated, while the AIA CCDs are backside illuminated.

HMI contains two identical CCD detectors, with a $4096^{2}$ pixel format, made by E2V. The CCDs have $12 \mu \mathrm{m}$ pixels and a full-well capacity of close to 200000 electrons. The $\mathrm{CCD}$ assemblies are mounted to the optics package using fiberglass insulator tubes in order to minimize the amount of heat transferred to them.

The CCDs are cooled passively using radiators mounted on top of the CCD assemblies, pointed perpendicular to the direction to the Sun. The expected CCD temperatures are below $-50 \mathrm{C}$, resulting in a negligible dark current. 


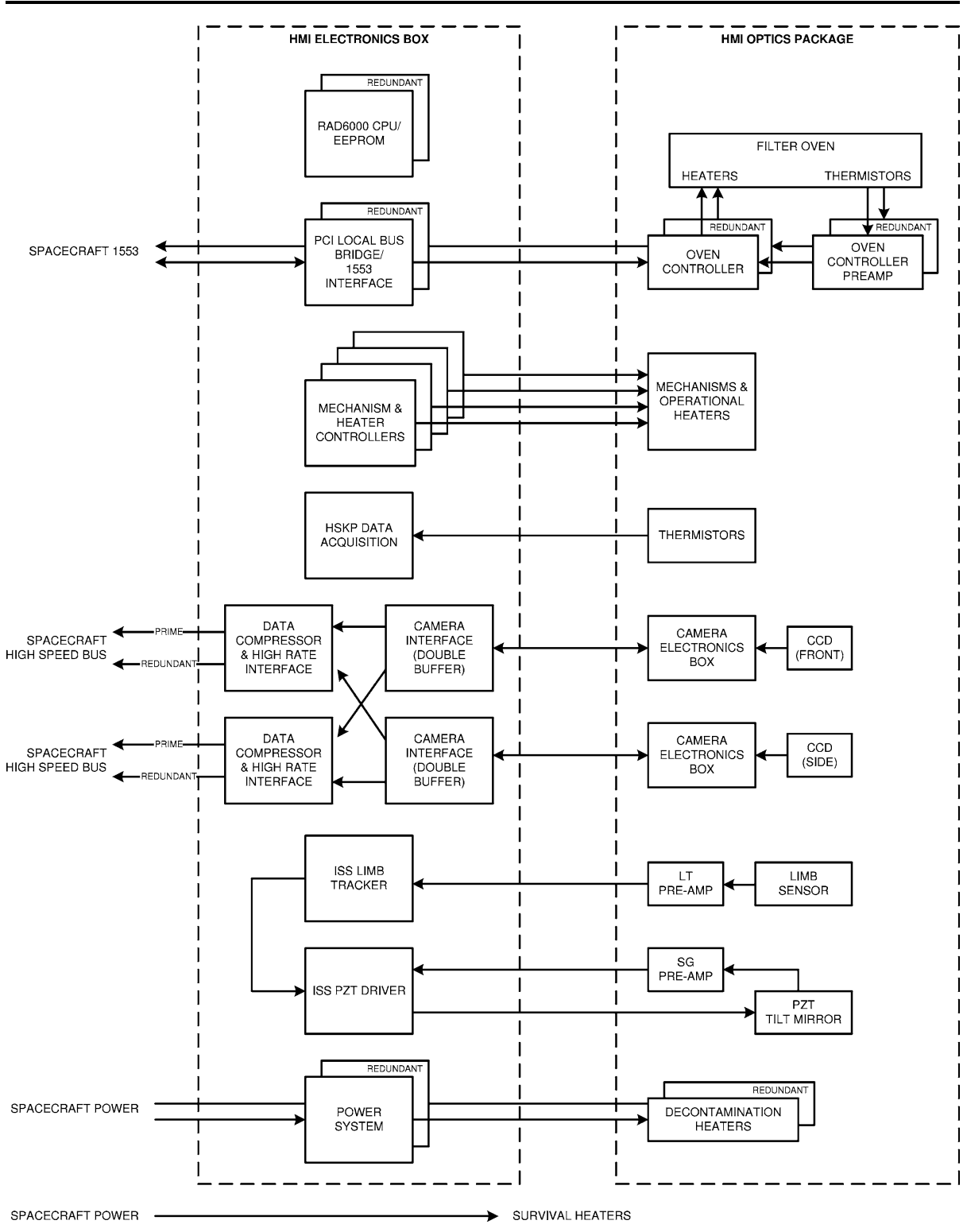

Figure 10 A simplified electronics block diagram of HMI. For simplicity the power and command-telemetry connections between the different components in the HEB have not been shown.

The cameras, made by Rutherford Appleton Laboratory (RAL), have a readout rate of 2 Mpixels s $^{-1}$ through each of four quadrant readout ports. Using all four amplifiers, a CCD can be read out with 14 bits per pixel in about 2.3 seconds. The actual performance is discussed in Wachter et al. (2011). In case of failures, it is also possible to read out using any two adjacent amplifiers or any single one, with correspondingly increased readout times, thus providing for a somewhat graceful degradation. 
In typical operation a programmable number of CCD rows (generally corresponding to well over a full image) are first shifted rapidly to eliminate any built-up charge, the shifting is stopped while the shutter is opened, and finally the CCD is read out. Generally the CCD is overscanned by one or two columns and rows in order to track performance.

\subsection{Data Processing Electronics}

The data from the CCDs are downlinked without any processing other than hardware compression and encoding. The different components of the electronics are described below.

\subsubsection{Camera Interface Board}

Each camera is connected to one CIF board using a spacewire (IEEE 1355) cable. Each CIF contains two image buffers, allowing one to be filled with new data while the other is being read out.

\subsubsection{Data Compression and High Rate Interface}

Next, the data are sent from the CIF boards to the combined Data Compression and High Rate Interface (DCHRI) boards. Normally each DCHRI board receives data from one CIF; however, the boards are cross-strapped allowing the data from both CIFs (and thus both cameras) to go to one DCHRI, thus providing significant redundancy.

The DCHRI boards do the following processing of the images (in order): crop (e.g. to not transmit data far above the solar limb), select which bits are to be used (e.g. to not transmit photon noise), put the resulting values through a lookup table (in order to better match the truncation to the photon noise at different intensities), and perform a lossless compression. The parameters of each of these steps can be set via loadable tables and can be different for each image in a framelist (see Section 2.11.6).

Each DCHRI connects to two separate redundant interfaces on the spacecraft data bus using spacewire links, thereby providing further redundancy. If these redundancies are used only a very small reduction in data rate results. The data rate allocated to HMI is $55 \mathrm{Mbit} \mathrm{s}^{-1}$, which may be adjusted by transmitting new tables to the spacecraft, with each DCHRI able to deliver in excess of the currently allocated rate.

\subsection{Flight Software}

The flight software (FSW) consists of several different modules. Some, e.g. those related to loading other modules, have little direct effect on the scientifically important aspects of the instrument. Others, such as the sequencer, are important for understanding the instrument capabilities. The following sections describe the scientifically important parts of the FSW.

\subsubsection{Overall Control}

The core of the FSW is a kernel running under a VxWorks real-time operating system. It is read from ROM at startup and is responsible for loading other modules and allocating CPU time for their execution.

Ordinarily the instrument is booted and loads the various modules from EEPROM into RAM. While not planned for routine use, it is possible to reload these parts of the software to the EEPROM. It is also possible to load new FSW directly to the RAM for testing or in case the EEPROM becomes non-functional.

In addition to being able to load the flight software itself, it is possible to load tables to memory for use by other modules, as described below. 


\subsubsection{Commanding Interface}

The FSW has the capability to receive and execute a large number of commands to load software and tables, control various subsystems such as mechanisms and heaters, configure various onboard control systems, initiate downloading of diagnostics data, and so forth.

It is also possible to execute simple onboard scripts. Typical examples of such scripts include ones to change the instrument heater settings during eclipses when the Earth blocks the Sun or ones to run different observing sequences during the eclipses or transits of the Moon or planets.

\subsubsection{Housekeeping}

The instrument provides a large variety of data in the housekeeping data stream. These include such items as temperatures, voltages, currents, limb-sensor voltages, states of subsystems, mechanism positions, command counts, and so forth. These are generally downlinked every four or eight seconds.

In addition, a copy of certain housekeeping values relevant to each image are also downlinked as a so-called Image Status Packet (ISP) with the image data. Currently a maximum of nine ISPs are allocated per 16 seconds, so although the cameras can run somewhat faster, this effectively sets the maximum frame rate at one image per 1.778 seconds.

The total allocated housekeeping data rate is $2353 \mathrm{bits} \mathrm{s}^{-1}$. Of this $1714 \mathrm{bits} \mathrm{s}^{-1}$ are currently allocated to the regular housekeeping and 612 bits s $^{-1}$ for the ISPs.

It is also possible to more intensively downlink selected hardware values at rates from $128 \mathrm{~Hz}$ to $512 \mathrm{~Hz}$ for about 3.5 minutes. This is typically used for sampling the ISS, but may also be used for such items as motor currents. These diagnostic data are downlinked over a separate channel, shared between various spacecraft subsystems, at a rate of $10 \mathrm{kbits} \mathrm{s}^{-1}$.

Finally, the instrument has a number of sensors connected directly to the spacecraft for health and safety monitoring when the instrument is not powered on.

\subsubsection{Thermal Control}

Each of the seven operational heaters are controlled by the FSW. In addition to being left constantly on or off, the FSW can run them at specified duty cycles using a pulse-width modulation scheme in which the heaters are on for $N$ time increments out of $M$, with $2 \leq$ $M \leq 100,0 \leq N \leq M$, and the time increments are fixed at $1 / 8$ second.

The FSW also provides basic thermostatic temperature control of the instrument. The scheme uses three temperatures: deadband low, target, and deadband high, and three duty cycles: cold, deadband, and off. The modes use the same $M$, but different $N$. At any given time, the heater zone is in one of three states: Rising state, in which the cold duty cycle is applied; Maintaining state, in which the deadband duty cycle is applied; and Dropping state, in which the duty cycle is 0 . The heater zone changes from Rising to Maintaining state when the target temperature is reached, and from Maintaining to Rising state if the temperature drops below the deadband low. If the temperature rises above the deadband high, the state is changed to Dropping. The state changes from Dropping to Maintaining if the temperature drops below the target.

The consequence of this control algorithm is that one has to either have a large deadband and accept that the temperature drifts with the environment, typically with a 24-hour period, or have a tight deadband and accept frequent, but smaller, changes in the temperatures as the duty cycles change. 


\subsubsection{Mechanism Control}

The mechanisms are also controlled by the FSW.

The front-door and alignment mechanisms are simple stepper motors and as such require little control. For the alignment mechanism, the FSW can seek for the zero crossing and, since there is no encoder, keep a count of steps taken. As mentioned earlier, the FSW limits the allowed positions to \pm 1500 steps from the center position.

The focus-mechanism and shutter interfaces are simple and require little active control (Sections 2.6.2 and 2.6.3).

The HCMs are substantially more complicated. While they can be operated by simple commanding to move clockwise or counterclockwise to a given target, two other features are implemented in the FSW. First, in order to minimize the time to perform a move, the FSW can be set to automatically choose the shorter direction. Second, since the stopping positions are inherently sensitive to such parameters as voltage and temperature, which are themselves somewhat variable, an optimization algorithm has been implemented. As discussed in Section 2.6.1, the HCMs use an adjustable delay to fine tune the stopping position. An encoder tells whether the motion overshot or undershot the position. The optimization algorithm uses this information to adjust the delay times up or down slightly to always end up near the desired position, regardless of temperature or voltage variations. Since the optimal delay also depends on move distance and to a lesser extent on the starting position, a separate delay is kept for each motor, start position, and stop position combination. A side effect of the use of this algorithm is that a given move has to be performed a number of times before the stopping position is accurate and it is thus desirable to perform changes in the set of tuning positions used during times when no sensitive data are being taken (e.g. during eclipses). Another side effect is that, since the delays are given as integer $\mu$ s (corresponding, roughly, to a part in $10^{6}$ of a rotation), the algorithm may introduce a small but systematic pattern in the stopping position. (Note that there was an error in this algorithm early in the ground testing and that data taken then need special correction. However, data taken 1 June 2007 and later should be free of this error.)

\subsubsection{Sequencer}

The part of the FSW most directly affecting the operation of the instrument from a scientific point of view is probably the sequencer. The sequencer is responsible for taking the images at the correct times and with the correct settings, as well as for executing calibrations at scheduled times.

The sequencer has two main levels of control: The top level determines which set of observations, described in standard framelists (see below), to execute at any given time, while the lower level schedules the individual frames.

At the top level, the sequencer uses a prioritized list to determine which framelist to execute next. By carefully selecting these priorities and the desired execution times for the different framelists, it is possible to interrupt the regular observables framelist to run a calibration sequence or to run different sequences depending on the time of day, for example.

The main control of the images taken is done by using so-called framelists. These contain, among other things, a list of relative times to take the individual filtergrams, as well as the wavelength, polarization, focus, exposure time, camera, and compression settings. At any given time only one framelist is executing. An example of a framelist and more information about what they contain are given in Appendix A.

Once the start time of a framelist is known, the exact time for each of the images can be calculated from the start time and the relative offset specified in the framelist. From these 
times, the time of execution of each instrument activity required to make an image can in turn be determined. Below is a list of the four main events scheduled during the taking of an image. The relative times of these are set by ground-commandable registers:

- Mechanism Move: This is the time at which the polarization selectors, wavelength selectors, and focus wheels start moving.

- Clear: This is the time at which the camera starts clearing the CCD and thereby initiates the image taking. See Section 2.9 for details.

- Shutter Open: This is the time at which the shutter opens.

- Readout: This is the time at which the readout of the image from the cameras to the CIF is initiated.

The events for the two cameras are independent, however a warning is issued if incompatible events overlap, e.g. if a HCM or focus wheel is moving between the start of the shutter move and the start of the readout.

\subsection{HMI-MDI Comparison}

To aid users of MDI in transitioning to use HMI, Table 2 compares key properties of the two instruments.

\section{Calibration}

Since HMI operates at a visible wavelength (unlike e.g. AIA) it was possible to perform a wide variety of end-to-end ground calibrations. We next describe the basic setups used for the calibration followed by a summary of the results obtained. Calibration details are described in separate articles for image quality and CCD performance (Wachter et al., 2011), for filter performance (Couvidat et al., 2011), and for polarization properties (Schou et al., 2010).

\subsection{Calibration Setups}

As described in Wachter et al. (2011) most ground calibrations were performed in one of two configurations. The most common setup uses a stimulus telescope that is a reverse of the HMI telescope. The stimulus telescope consists of primary and secondary lenses manufactured to the same specifications as the flight optics. As a result there is significant field curvature in the stimulus telescope.

The stimulus telescope can be fed with either white light from a stabilized lamp or with laser light. The lamp light is fed into a fiber bundle that is then imaged onto the pupil using a condenser lens. This setup scrambles the light and ensures a uniform illumination of both the image and pupil. At the focus of the stimulus telescope it is possible to mount a number of different targets, most of which are made with a metal film deposited on a glass slide. The stimulus telescope focus can be found in auto-collimation using a large optical flat.

The same basic setup is used with one of two laser light sources. A tunable dye laser is fed through an optical fiber from another room and typically illuminates a rotating diffuser. A tunable solid-state laser was used for some tests. The solid-state laser mounts directly on the stimulus telescope and uses a simple diffuser. Unfortunately the solid-state laser is not very bright, which limited its use for calibrations.

The other main setup feeds sunlight to HMI. A heliostat on the roof tracks the Sun and directs sunlight through a tube to a fold mirror in front of the instrument. 
Table 2 Comparison of MDI and HMI. For MDI: FD refers to full-disk mode, HR to high-resolution mode, and structure to the continuous data. Note that some numbers for HMI (e.g. the cadences and filtergram positions) are nominal and may be changed as operational experience is gained.

\begin{tabular}{|c|c|c|}
\hline Property & MDI & HMI \\
\hline Target line & Ni I $6768 \AA$ & Fe I $6173 \AA$ \\
\hline Aperture & $12.5 \mathrm{~cm}$ & $14.0 \mathrm{~cm}$ \\
\hline Optical resolution $(\lambda / \mathrm{D})$ & $1.17 \operatorname{arcsec}$ & $0.91 \operatorname{arcsec}$ \\
\hline Pixel size & $\begin{array}{l}1.98 \operatorname{arcsec}(\mathrm{FD}) \\
0.61 \operatorname{arcsec}(\mathrm{HR})\end{array}$ & $0.505 \operatorname{arcsec}$ \\
\hline $\mathrm{CCD}$ & One $1024 \times 102421 \mu \mathrm{m}$ & Two $4096 \times 409612 \mu \mathrm{m}$ \\
\hline Front window FWHM & $50 \AA$ & $50 \AA$ \\
\hline Blocking filter FWHM & $8 \AA$ & $8 \AA$ \\
\hline Lyot design & $1: 1: 2: 2: 4: 8$ & $1: 2: 4: 8: 16$ \\
\hline Untuned FWHM & $465 \mathrm{~m} \AA$ & $612 \mathrm{~m} \AA$ \\
\hline Tunable element FWHMs & $\begin{array}{l}94 \text { mÅ Michelson } \\
189 \text { mÅ Michelson }\end{array}$ & $\begin{array}{l}86 \text { mÅ Michelson } \\
172 \mathrm{~m} \AA \text { Michelson } \\
344 \mathrm{~m} \AA \text { Lyot }\end{array}$ \\
\hline $\begin{array}{l}\text { Final filter FWHM } \\
\text { Spectral resolution }\end{array}$ & $85 \mathrm{~m} \AA$ & $76 \mathrm{~m} \AA$ \\
\hline$(\lambda / F W H M / 1000)$ & 80 & 81 \\
\hline Polarization & $I \pm Q$ and $I \pm V$ & All \\
\hline Filtergram cadence & 3 seconds & $\begin{array}{l}3.75 \text { seconds per camera } \\
1.875 \text { overall }\end{array}$ \\
\hline Filtergram positions & 5 at $75 \mathrm{~m} \AA$ spacing & 6 at $69 \mathrm{~m} \AA$ spacing \\
\hline Nominal observables cadence & 60 seconds & 45 seconds \\
\hline Data rate & $\begin{array}{l}160 \mathrm{kbit} \mathrm{s}^{-1}(\mathrm{FD} \text { and HR}) \\
5 \mathrm{kbit} \mathrm{s}^{-1} \text { (Structure) }\end{array}$ & $55 \mathrm{Mbits}^{-1}$ \\
\hline
\end{tabular}

The stimulus telescope, as used for image-quality measurements, as well as the overall calibration setup are described in more detail by Wachter et al. (2011). Uses for filter characterization are described by Couvidat et al. (2011), as is the use of the heliostat.

Tests were performed in two different environments: In one case the instrument resides in a vacuum tank with an optical-quality window. In the vacuum tank the CCDs could be cooled to dramatically reduce the dark current and thus photon noise. However the optical and polarization characteristics of the window are not known reliably. A sophisticated temperature control system was constructed to perform thermal tests on the instrument.

In the other case the instrument is operated in a clean tent. This is more convenient, but has disadvantages of its own. At room temperature there is a significant and often variable amount of dark current, requiring compensation in the analysis. The presence of air in the instrument changes the focus dramatically. To compensate for this, an air-to-vacuum corrector consisting of a plano-concave and plano-convex lens pair with identical radius of curvature and spaced by an adjustable amount is placed between the stimulus telescope and the instrument. Alternatively, the targets in the stimulus telescope can be moved a very specific distance from the position determined by the auto-collimation. Finally, the tuning of the Michelsons changes by an amount that depends on air pressure, temperature, and humidity. 
Since the stimulus telescope is in either case in air, the presence of air currents causes small image distortions and degradation of the image quality.

Standard calibration sequences were developed for the Comprehensive Performance Tests (CPTs) which were performed regularly before launch to monitor the performance of the instrument during integration and testing. The instrument orientation changed $90^{\circ}$ in roll around the line-of-sight direction, with respect to the stimulus telescope as well to the direction of gravity, when it was moved from LMSAL in Palo Alto, where the instrument was assembled and tested, to NASA Goddard Space Flight Center (GSFC), where the integration to the spacecraft was performed. The orientation at Astrotech Science Operations in Florida, where the spacecraft was prepared for launch and some of the tests preformed, was the same as at GSFC.

Finally, it should be noted that some of the calibrations have been deferred to on-orbit for practical reasons.

\subsection{Image Quality}

Details of the image-quality calibration setup, procedures, and results are given by Wachter et al. (2011). Some of the main results are given below. The Strehl ratio is measured to be $0.74 \pm 0.03$ or better. The uncertainty is due to difficulties with compensating for the test setup, and is thus expected to be better on orbit. Since a wavefront error in the front window was polished out, thereby substantially improving the Modulation Transfer Function (MTF) (Wachter et al., 2011), no measurable changes of the MTF have been observed during the CPTs and other optical-performance tests.

The optical distortion has been modeled with Zernike polynomials and is as large as two pixels at the edge of the field of view. Residuals are of the order of a few hundredths of a pixel, and are dominated by irregularities in the CCD lattice.

Residual image motions of up to half a pixel are induced by the rotation of the HCMs. These motions have been consistently observed, and are corrected for in the observables calculations.

The performance of the ISS is shown in Figure 11. As can be seen, the attenuation is significant over a wide frequency range.

Small measured variations in the best focus across the field of view can be expressed as a field curvature ( $0.4 \mathrm{~mm}$ focal-plane variation from center to edge) and a field tilt (up to $0.5 \mathrm{~mm}$ focal-plane variation from edge to edge). Given that the HMI focus steps are 1 $\mathrm{mm}$, corresponding to less than a depth of focus, this additional variation does not degrade the image quality significantly. The best focus has been set near the middle of the available focus range (focus position 9 in a range from 1 through 16), with a bias toward a higher focus position, because adjustment of the heaters at the edge of the front window can only bring the best focus to a lower position.

The two HMI cameras are aligned (with a residual shift of less than ten pixels), and the shift and the rotation $\left(0.08^{\circ}\right)$ have been monitored. During the calibration period, the cameras have shifted slightly (by a couple of pixels) relative to each other, possibly due to changing mechanical stresses in the instrument. Small shifts are not a concern because they can be constantly monitored by tracking the solar limb or correlating the images.

The flat field shows the expected structure originating in the design of the CCD, contamination in the optics, and large-scale optical variations. The internal vignetting (see Section 2.4.3) results in a sharp drop off in the flat field at the outer edge of the field of view. The large-scale flat field could be determined with an accuracy of $0.2 \%$. Drifts observed in the small-scale flat field in vacuum are believed to be caused by outgassing material condensing 
Figure 11 Example of the ratio of the observed signal on the limb sensors (sampled at a high rate, as described in Section 2.5) with and without the ISS turned on for a variety of frequencies. Also shown is a model of the response. Both the measured data and the model depend on the instrument gain settings.

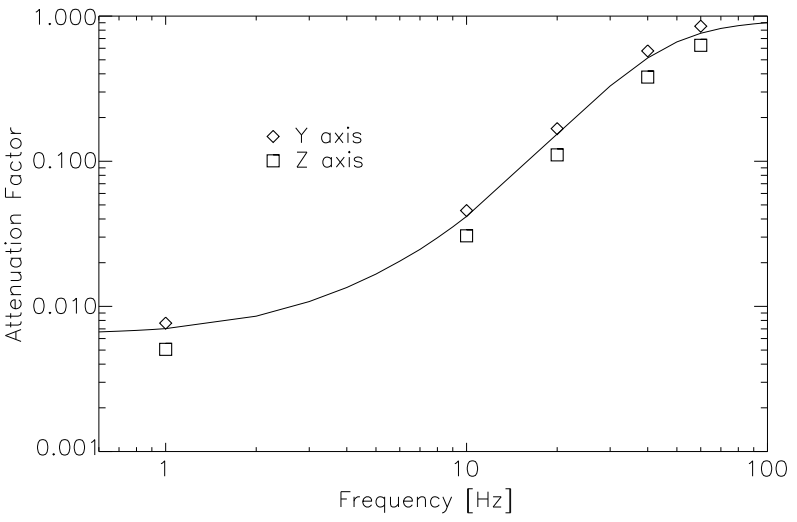

on the cold CCDs. Given the lengthy outgassing period planned during on-orbit commissioning, during which the CCDs are kept warmer than the rest of the instrument, and the ability to drive any condensed material off by warming the $\mathrm{CCD}$, the amount of material to condense is expected to be small in space. Small-scale flat-field monitoring will be performed to keep the flat field up to date at the aforementioned level of accuracy (Wachter and Schou, 2009).

The CCD full well is around $12000 \mathrm{DN}$, which with an inverse gain of roughly $16 e^{-} \mathrm{DN}^{-1}$ corresponds to roughly $200 \mathrm{k} e^{-}$. The camera non-linearity is better than about $1 \%$ up to the point where the system saturates. Since the exposure time can be controlled accurately over a large range, it is expected that the maximum exposure level near disk center will be close to full well, but the determination of the exact target has been deferred to commissioning.

The dark current is negligible when the CCDs are operated at their design temperature. The read noise is negligible compared to the photon noise.

\subsection{Wavelength Dependence}

The instrument also underwent a significant calibration effort in the area of filter performance. This is described in detail by Couvidat et al. (2011) and summarized below.

Observable sequences taken by HMI to measure the Doppler velocity and complete magnetic-field vector at the solar surface consist of full-disk filtergrams observed at six wavelengths spanning the range $\pm 172.5 \mathrm{~m} \AA$ around the target wavelength of $6173 \AA$. In order to accurately derive the Doppler velocities and vector magnetic fields from these six wavelengths and various polarization states, the transmission profiles of all the components of the HMI optical and filter system described in Section 2.4 need to be known: this is the purpose of the wavelength-dependence calibration.

The five stages of the Lyot filter and the two Michelson interferometers have transmission profiles, which can be fully characterized, in a first approximation, by only three parameters: the FSR, the relative phases, and the contrasts (ranging from 0 to 1 ). Both the phase and contrast vary across the element aperture and therefore are given as $2 \mathrm{D}$ maps. The FSRs of the elements are, to a very good approximation, equal to twice their FWHM. To determine these parameters we took cotune and detune sequences with various light sources. A detune sequence is a series of 27 or $31 \mathrm{HMI}$ images for which the three wavelength selectors are tuned so that the peaks of maximum transmittance of the tunable elements do not necessarily coincide. For a cotune sequence the tunable elements are tuned together. 
From a detune sequence with laser light, the phase and contrast maps of the three tunable elements can be determined. Such maps can be obtained both in obsmode and calmode. Detune sequences taken at different laser wavelengths give us access to the FSRs of the tunable elements, and also the phase and contrast maps of the non-tunable elements (Lyot elements E2 to E5). The FSRs of the non-tunable elements and the transmission profiles of the front window and blocking filters have to be measured separately while these parts are outside the instrument. Detune sequences in sunlight also allow a determination of the phases of the tunable elements. In sunlight, the contrasts of these elements cannot be assessed and the phases can only be derived in calmode. We fit for the phases of the tunable elements as well as the parameters defining the Fe I solar line (approximated by a Gaussian profile for convenience). Numerous detune sequences taken on the ground showed the high quality of the HMI filter elements. The phase ranges of the three tunable elements are relatively small (less than $20^{\circ}$ ), and their contrasts are very high ( $>0.95$ on average), especially compared to the Michelson interferometers in the MDI instrument. Their FSRs are well within specifications. For reasons that are not fully understood, the average phases of the tunable elements are slightly different in obsmode and calmode (especially for E1). This is partly due to the non-negligible angular dependence of these elements. Similarly, for the narrowband Michelson there seems to be a small difference between phase maps obtained from the front and side cameras. The overall mean transmission profile of the non-tunable elements is centered around $+16 \mathrm{~m} \AA$ from the target wavelength, with a FWHM of $\approx 624.5 \mathrm{~m} \AA$ for the main peak. Two small main sidelobes are located at about $\pm 1 \AA$.

The best estimates of the FWHM of the various elements are:

- Front window: $48.5 \AA$

- Blocking filter: $8.43 \AA$

- Lyot element E2: $0.704 \AA$

- Lyot element E3: $1.390 \AA$

- Lyot element E4: $2.84 \AA$

- Lyot element E5: $5.68 \AA$

- Lyot element E1: $346.75 \mathrm{m \AA} \pm 2.3 \mathrm{~m} \AA$ (tuned)

- Wide Band (WB) Michelson: $170.95 \mathrm{~m} \AA \pm 0.8 \mathrm{~m} \AA$ (tuned)

- Narrow Band (NB) Michelson: $85.45 \mathrm{~m} \AA \pm 0.45 \mathrm{~m} \AA$ (tuned)

- Untuned part: $624.5 \mathrm{m \AA}$

- Complete filter: $76.15 \mathrm{mÅ}$ (at center tuning position)

In some cases uncertainties are given, for others no good estimates are available, although the Lyot element uncertainties are likely less than $1 \%$.

The blocking filter and front window are composed of multiple glass blocks that unfortunately act as weak Fabry-Pérot interferometers because of partial reflections at the glass interfaces. This produces a fringe pattern visible in the filtergrams and in the phase and contrast maps of the filter elements. The fringe pattern can be characterized by taking detune sequences in white light (from a lamp) in calmode and obsmode. The transmission profile of the non-tunable part of the HMI optical filter can be expanded into a Fourier series. The first seven Fourier coefficients are accessible through this analysis. The Fourier-coefficient maps at these different spatial frequencies show the different fringe patterns and allow us to pinpoint their origin. An issue is that the phases of these patterns depend on the temperature of the front window and blocking filter. Therefore these fringes will not be stable for some time (with an e-folding time similar to the roughly ten to fifteen minutes for the focus stabilization) after each daily eclipse during the Spring and Fall eclipse seasons (each of which lasts roughly 21 days). 
The detailed wavelength profiles of the blocking filter and front window also means that each detune- or cotune-sequence position does not receive exactly the same amount of incident light: the integral value of the transmission profiles varies, resulting in an I-ripple. Detune sequences in white light allow us to measure the overall I-ripple of HMI. Fine-tuning sequences in which each element is tuned separately allow us to measure the individual $I$-ripples of the tunable elements. These individual $I$-ripples are $<1 \%$ (relative peak-to-peak variation of transmitted intensity), while the overall $I$-ripple is slightly below $2 \%$. The $I$ ripple was not taken into account in the analysis.

Transmission profiles, fringe patterns, and I-ripples may depend on the angle of incidence of light rays, and on the temperature of the optical-filter elements. Hence the need to characterize these angular and temperature dependencies. The angular dependence of the tunable elements is obtained in calmode from detune sequences taken by moving a small field stop to various positions in the image plane. The same can be done in obsmode by moving an aperture stop across the front window. The Lyot element E1 is the tunable element with the largest angular dependence, followed by the NB and the WB Michelsons. This was expected, as the angular dependence of a wide-field Lyot element varies as $\theta^{2}$ (where $\theta$ is the angle of incidence) while it varies as $\theta^{4}$ for a wide-field Michelson interferometer. E1 also seems to show a significant amount of azimuthal dependence. The temperature dependence of the tunable elements is satisfactory. Indeed, the wavelength drift is only about $15 \mathrm{~m}^{-1}$ at $30 \mathrm{C}$. Fortunately the $I$-ripple does not vary with temperature or with a change of focus blocks.

Finally, the overall throughput (ratio of transmitted over input intensity) of HMI was measured at $\approx 1.35 \%$, which is more than adequate for the 45 -second cadence of the observable sequence.

An example of the resulting six tuning position transmission profiles is shown in Figure 6, with respect to the Fe I solar line profile at rest and at disk center (R.K. Ulrich, private communications).

After launch the orbital velocity of SDO may be used to improve the instrument calibration on orbit. The Sun-SDO radial velocity is known with an exquisite precision that allows us, in theory, to reach a much better accuracy on the FSRs of the tunable elements than from ground tests, and to reduce some uncertainties in the filter transmission profiles. However, due to the limited range of this radial velocity $\left( \pm 3.5 \mathrm{~km} \mathrm{~s}^{-1}\right.$ from the orbit), it is not clear to what extent the calibration can be improved. This will be further investigated after launch.

As an illustration of the end-to-end performance, Figure 12 shows the expected photon noise in the Dopplergrams.

\subsection{Polarization}

The final major calibration area is polarization. The details of this effort are described in Schou et al. (2010).

For the polarization tests, a Polarization Calibration Unit (PCU), described by Schou et al. (2010), is inserted into the beam between the stimulus telescope and HMI. The PCU allows for a polarizer and an approximately quarter-wave plate to be inserted in the beam, each of which can be rotated to arbitrary angles.

By taking a carefully crafted set of images with various settings of the PCU and of the HMI polarization selectors, it is possible to derive almost all of the relevant properties of the stimulus telescope, PCU, front window, and polarization selectors. The undetermined parameters (polarization-angle zero point and one of the polarization-selector rotation angles) were determined from independent measurements. 
Figure 12 Expected Doppler noise from photon statistics calculated from the results of the wavelength calibration and averaged over azimuth. Results are shown for a variety of line-of-sight velocities. The variation with azimuth is small relative to the variation with velocity.

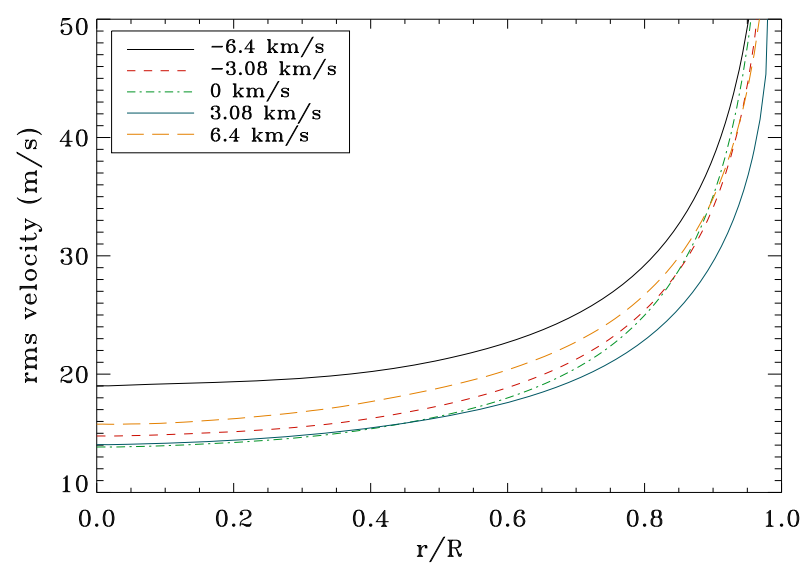

From these measurements it is in turn possible to determine a model of the response of the instrument to a given input Stokes vector. The main results are that the polarimetric model indicates that the crosstalk, after calibration, between $Q, U$, and $V$ is less than $1 \%$ across the FOV and that the leakage from $Q$ and $U$ to $V$ without calibration is below $5 \%$, both meeting the requirements.

Some of the terms in the calibration, in particular the $I$ to $Q, U$, and $V$ leakages (telescope polarization) are difficult to determine using ground-based measurements, but are straightforward to determine on orbit, and the determination of those terms have thus been deferred. It may be noted that apart from these terms it is generally difficult to do a proper polarization calibration on orbit as there are no well-defined polarization sources available.

Several of the parameters describing the polarimetric model have a significant temperature dependence, but it has also proven possible to model this with sufficient accuracy to meet specifications.

\section{Conclusion}

The HMI instrument provides full-disk solar imaging at one arcsecond resolution with unprecedented temporal coverage and resolution of both line-of-sight observables and full Stokes parameters.

The instrument was designed based on the highly successful MDI instrument, incorporating improvements in several areas of the optics and electronics. Among the improvements the substantial redundancy means that the instrument will be able to continue to provide high-quality data, even in case of the malfunction of significant components.

The instrument has gone through a substantial calibration effort to be able to provide reliable inferences about the Sun. We have been able to characterize all relevant aspects of the instrument with a very good accuracy.

Given the above we are confident that the instrument will provide results of unprecedented quality for a long period of time.

Updated documentation about the instrument will be available from the project website http://hmi.stanford.edu and the observables from the instrument will be available from http://jsoc.stanford.edu shortly after the observations are taken. 
Acknowledgements A large number of people in the Stanford Solar Physics group provided invaluable help during the design and test of the instrument, including: Jim Aloise, Art Amezcua, John Beck, KehCheng Chu, Carl Cimilluca, Romeo Durscher, Sarah Gregory, Tim Larson, Kim Ross, Jeneen Sommers, and Hao Thai. We would also like to thank Robert Byer, Carsten Langrock, Joe Schaar, and Karel Urbanek at Stanford's Ginzton Laboratory for help with designing and assembling the tunable solid-state laser. At LMSAL a large number of engineers and other personnel helped with design and test, including Ron Baraze, Jerry Drake, Dexter Duncan, Jay Dusenbury, Chris Edwards, Barbara Fischer, Glen Gradwohl, Gary Heyman, Noah Katz, Dwana Kacensky, Dave Kirkpatrick, Gary Kushner, Russ Lindgren, Gary Linford, Dnyanesh Mathur, Edward McFeaters, Keith Mitchell, Rose Navarro, Tom Nichols, Roger Rehse, J.-P. Riley, Larry Springer, Bob Stern, Louie Tavarez, Edgar Thomas, Darrel Torgerson, Ross Yamamoto, Carl Yanari, and Kent Zickhur. We also thank Tom Anderson, Lisa Bartusek, Michael Bay, Liz Citrin, Peter Gonzales, Juli Lander, Eliane Larduinat, Wendy Morgenstern, Dean Pesnell, Chad Salo, Mike Scott, and Barbara Thompson at GSFC, Juan Manuel Borrero, Greg Card, Rebecca Centeno, Tony Darnell, and Bruce Lites at HAO, KD Leka at NWRA/CORA, Matthew Clapp, Sarah Dunkin, and Nick Waltham at RAL, Gary Auker and Rob Wilson at E2V, John Hunter, Ian Miller, and Jeff Wimperis at LightMachinery, and Gerard Gleeson at ASO. Finally we would like to thank Jack Harvey for useful comments and advice. This work was supported by NASA contract NAS5-02139 to Stanford University. The HMI data used are courtesy of NASA/SDO and the HMI science team.

Open Access This article is distributed under the terms of the Creative Commons Attribution Noncommercial License which permits any noncommercial use, distribution, and reproduction in any medium, provided the original author(s) and source are credited.

\section{Appendix A: Sequencer and Framelist Examples}

Table 3 shows an example of a framelist. In order, the columns are the following:

- FID: The Frame ID is simply downlinked with the image and has no effect on the sequencer execution. In the present case it is used to indicate the position in the line and the polarization state. It can also be used to indicate frames intended for calibration only.

- RelTime: This is the time (in ms) of the frame relative to the start time of the sequence.

- Img: The type of readout (1, 2, or 4 port), cropping and compression used.

- PL: The index of the desired polarization setting.

- WL: The index of the wavelength setting desired.

- CF: The focus position used. 1 - 16 indicate normal images. 17 calmode.

- Exp: The index of the exposure time desired.

- Obspath: The camera used. IMAGE indicates that an exposure is taken. DARK indicates that a dark frame is taken.

Note that most of the settings use an index into another table. That table then details the settings of the individual mechanisms. In many cases a default value is specified. The corresponding values are kept separately in registers in the FSW. This allows for changing parameters such as the focus position and exposure time without remaking framelists.

Similarly PL positions 258 and 259 correspond to LCP and RCP while 250 through 253 are four positions allowing for the determination of $I, Q, U$, and $V$.

In the example shown in Table 3 , WL positions 465, 467, 469, 471, 473, and 475 correspond, in order, to $\mathrm{I} 5, \mathrm{I} 4, \mathrm{I} 3, \mathrm{I} 2$, I1, and $\mathrm{I} 0$, which in turn correspond to increasing wavelength.

As can be seen, the framelist loops twice through the wavelengths in a particular nonsequential order I3, I4, I0, I5, I1, I2. Combined with choosing the starting point such that the center wavelengths (I2 and I3) are centered on the target times ( 0 seconds and 45 seconds), this minimizes the errors in the inferred Doppler velocities. 
Table 3 Framelist taking LOS data on the front and full IQUV on the side camera. See text for details.

\begin{tabular}{|c|c|c|c|c|c|c|c|}
\hline FID & RelTime & Img & PL & WL & $\mathrm{CF}$ & Exp & ObsPath \\
\hline 10098 & 0 & DEFAULT & 258 & 469 & DEFAULT & DEFAULT & FRONT2_IMAGE \\
\hline 10090 & 1875 & DEFAULT & 250 & 469 & DEFAULT & DEFAULT & SIDE1_IMAGE \\
\hline 10099 & 3750 & DEFAULT & 259 & 469 & DEFAULT & DEFAULT & FRONT2_IMAGE \\
\hline 10091 & 5625 & DEFAULT & 251 & 469 & DEFAULT & DEFAULT & SIDE1_IMAGE \\
\hline 10079 & 7500 & DEFAULT & 259 & 467 & DEFAULT & DEFAULT & FRONT2_IMAGE \\
\hline 10070 & 9375 & DEFAULT & 250 & 467 & DEFAULT & DEFAULT & SIDE1_IMAGE \\
\hline 10078 & 11250 & DEFAULT & 258 & 467 & DEFAULT & DEFAULT & FRONT2_IMAGE \\
\hline 10071 & 13125 & DEFAULT & 251 & 467 & DEFAULT & DEFAULT & SIDE1_IMAGE \\
\hline 10158 & 15000 & DEFAULT & 258 & 475 & DEFAULT & DEFAULT & FRONT2_IMAGE \\
\hline 10150 & 16875 & DEFAULT & 250 & 475 & DEFAULT & DEFAULT & SIDE1_IMAGE \\
\hline 10159 & 18750 & DEFAULT & 259 & 475 & DEFAULT & DEFAULT & FRONT2_IMAGE \\
\hline 10151 & 20625 & DEFAULT & 251 & 475 & DEFAULT & DEFAULT & SIDE1_IMAGE \\
\hline 10059 & 22500 & DEFAULT & 259 & 465 & DEFAULT & DEFAULT & FRONT2_IMAGE \\
\hline 10050 & 24375 & DEFAULT & 250 & 465 & DEFAULT & DEFAULT & SIDE1_IMAGE \\
\hline 10058 & 26250 & DEFAULT & 258 & 465 & DEFAULT & DEFAULT & FRONT2_IMAGE \\
\hline 10051 & 28125 & DEFAULT & 251 & 465 & DEFAULT & DEFAULT & SIDE1_IMAGE \\
\hline 10138 & 30000 & DEFAULT & 258 & 473 & DEFAULT & DEFAULT & FRONT2_IMAGE \\
\hline 10130 & 31875 & DEFAULT & 250 & 473 & DEFAULT & DEFAULT & SIDE1_IMAGE \\
\hline 10139 & 33750 & DEFAULT & 259 & 473 & DEFAULT & DEFAULT & FRONT2_IMAGE \\
\hline 10131 & 35625 & DEFAULT & 251 & 473 & DEFAULT & DEFAULT & SIDE1_IMAGE \\
\hline 10119 & 37500 & DEFAULT & 259 & 471 & DEFAULT & DEFAULT & FRONT2_IMAGE \\
\hline 10110 & 39375 & DEFAULT & 250 & 471 & DEFAULT & DEFAULT & SIDE1_IMAGE \\
\hline 10118 & 41250 & DEFAULT & 258 & 471 & DEFAULT & DEFAULT & FRONT2_IMAGE \\
\hline 10111 & 43125 & DEFAULT & 251 & 471 & DEFAULT & DEFAULT & SIDE1_IMAGE \\
\hline 10098 & 45000 & DEFAULT & 258 & 469 & DEFAULT & DEFAULT & FRONT2_IMAGE \\
\hline 10092 & 46875 & DEFAULT & 252 & 469 & DEFAULT & DEFAULT & SIDE1_IMAGE \\
\hline 10099 & 48750 & DEFAULT & 259 & 469 & DEFAULT & DEFAULT & FRONT2_IMAGE \\
\hline 10093 & 50625 & DEFAULT & 253 & 469 & DEFAULT & DEFAULT & SIDE1_IMAGE \\
\hline 10079 & 52500 & DEFAULT & 259 & 467 & DEFAULT & DEFAULT & FRONT2_IMAGE \\
\hline 10072 & 54375 & DEFAULT & 252 & 467 & DEFAULT & DEFAULT & SIDE1_IMAGE \\
\hline 10078 & 56250 & DEFAULT & 258 & 467 & DEFAULT & DEFAULT & FRONT2_IMAGE \\
\hline 10073 & 58125 & DEFAULT & 253 & 467 & DEFAULT & DEFAULT & SIDE1_IMAGE \\
\hline 10158 & 60000 & DEFAULT & 258 & 475 & DEFAULT & DEFAULT & FRONT2_IMAGE \\
\hline 10152 & 61875 & DEFAULT & 252 & 475 & DEFAULT & DEFAULT & SIDE1_IMAGE \\
\hline 10159 & 63750 & DEFAULT & 259 & 475 & DEFAULT & DEFAULT & FRONT2_IMAGE \\
\hline 10153 & 65625 & DEFAULT & 253 & 475 & DEFAULT & DEFAULT & SIDE1_IMAGE \\
\hline 10059 & 67500 & DEFAULT & 259 & 465 & DEFAULT & DEFAULT & FRONT2_IMAGE \\
\hline 10052 & 69375 & DEFAULT & 252 & 465 & DEFAULT & DEFAULT & SIDE1_IMAGE \\
\hline 10058 & 71250 & DEFAULT & 258 & 465 & DEFAULT & DEFAULT & FRONT2_IMAGE \\
\hline 10053 & 73125 & DEFAULT & 253 & 465 & DEFAULT & DEFAULT & SIDE1_IMAGE \\
\hline 10138 & 75000 & DEFAULT & 258 & 473 & DEFAULT & DEFAULT & FRONT2_IMAGE \\
\hline 10132 & 76875 & DEFAULT & 252 & 473 & DEFAULT & DEFAULT & SIDE1_IMAGE \\
\hline 10139 & 78750 & DEFAULT & 259 & 473 & DEFAULT & DEFAULT & FRONT2_IMAGE \\
\hline 10133 & 80625 & DEFAULT & 253 & 473 & DEFAULT & DEFAULT & SIDE1_IMAGE \\
\hline 10119 & 82500 & DEFAULT & 259 & 471 & DEFAULT & DEFAULT & FRONT2_IMAGE \\
\hline 10112 & 84375 & DEFAULT & 252 & 471 & DEFAULT & DEFAULT & SIDE1_IMAGE \\
\hline 10118 & 86250 & DEFAULT & 258 & 471 & DEFAULT & DEFAULT & FRONT2_IMAGE \\
\hline 10113 & 88125 & DEFAULT & 253 & 471 & DEFAULT & DEFAULT & SIDE1_IMAGE \\
\hline
\end{tabular}


In both halves of the framelist, the front camera does LCP and RCP at each wavelength, thereby allowing for the Doppler and LOS field to be obtained. The side camera, on the other hand, does two of the four polarizations in the first half and the other two in the second half, thereby allowing for a 90 -second cadence using data from that camera only.

Many other framelists with various tradeoffs are, of course, possible. Apart from ones with different details in the regular observing sequences, they include ones taking detunes, focus sweeps, linearity data, and so forth.

\section{Appendix B: Electronic Supplementary Material}

\section{B.1. Optical Prescription}

For reference two Zemax optical prescriptions are included as supplementary material. Both files are for the instrument as designed and do not include any known or suspected aberrations. Polarization has not been included.

The first model (HMI_FOLD_2005-05-19.ZMX and the corresponding text prescription HMI_FOLD_2005-05-19.txt (supplementary text)) has configurations for the various focus settings and calmode. For clarity it does not include the final fold mirrors below the CCDs.

The second model (HMI_FOLD_2005-05-19_paths.ZMX and the corresponding text prescription HMI_FOLD_2005-05-19_paths.txt (supplementary text)) is for best focus only, but includes the alternate paths through the Michelsons, the path to the second camera, and the final fold mirrors.

None of the models include the path to the limb sensor.

\section{B.2. Heater Zones and Thermistor Locations}

For reference, the placement of the various thermistors is shown in the file thermistors.pdf (supplementary pdf). The heaters zones and heaters are included as HEATERS.PDF (supplementary pdf).

\section{References}

Couvidat, S., Schou, J., Shine, R.A., Bush, R.I., Miles, J.W., Scherrer, P.H., Rairden, R.L.: 2011, Wavelength dependence of the Helioseismic and Magnetic Imager (HMI) instrument onboard the Solar Dynamics Observatory (SDO). Solar Phys. doi:10.1007/s11207-011-9723-8.

Lemen, J.R., Duncan, D.W., Edwards, C.G., Friedlaender, F.M., Jurcevich, B.K., Morrison, M.D., Springer, L.A., Stern, R.A., Wuelser, J., Bruner, M.E., Catura, R.C.: 2004, The solar X-ray imager for GOES. In: Fineschi, S., Gummin, M.A. (eds.) SPIE 5171, 65-76. doi:10.1117/12.507566.

Lemen, J.R., Title, A.M., Akin, D.J., Boerner, P.F., Chou, C., Drake, J.F., Duncan, D.W., Edwards, C.G., Friedlaender, F.M., Heyman, G.F., Hurlburt, N.E., Katz, N.L., Kushner, G.D., Levay, M., Lindgren, R.W., Mathur, D.P., McFeaters, E.L., Mitchell, S., Rehse, R.A., Schrijver, C.J., Springer, L.A., Stern, R.A., Tarbell, T.D., Wuelser, J.P., Wolfson, C.J., Yanari, C., Bookbinder, J.A., Cheimets, P.N., Caldwell, D., Deluca, E.E., Gates, R., Golub, L., Park, S., Podgorski, W.A., Bush, R.I., Scherrer, P.H., Gummin, M.A., Smith, P., Auker, G., Jerram, P., Pool, P., Soufli, R., Windt, D.L., Beardsley, S., Clapp, M., Lang, J., Waltham, N.: 2011, The Atmospheric Imaging Assembly (AIA) on the Solar Dynamics Observatory (SDO). Solar Phys. doi:10.1007/s11207-011-9776-8.

Norton, A.A., Graham, J.P., Ulrich, R.K., Schou, J., Tomczyk, S., Liu, Y., Lites, B.W., López Ariste, A., Bush, R.I., Socas-Navarro, H., Scherrer, P.H.: 2006, Spectral line selection for HMI: A comparison of Fe I $6173 \AA$ and Ni I 6768 Å. Solar Phys. 239, 69-91. doi:10.1007/s11207-006-0279-y. 
Scherrer, P.H., Bogart, R.S., Bush, R.I., Hoeksema, J.T., Kosovichev, A.G., Schou, J., Rosenberg, W., Springer, L., Tarbell, T.D., Title, A., Wolfson, C.J., Zayer, I., MDI Engineering Team: 1995, The solar oscillations investigation-Michelson Doppler Imager. Solar Phys. 162, 129-188. doi:10.1007/BF00733429.

Scherrer, P.H., Schou, J., Bush, R.I., Kosovichev, A.G., Bogart, R.S., Hoeksema, J.T., Liu, Y., Duvall, T.L., Title, A.M., Tomczyk, S., HMI Science Team: 2011, The helioseismic and magnetic imager investigation. Solar Phys. doi:10.1007/s11207-011-9834-2.

Schou, J., Borrero, J.M., Norton, A.A., Tomczyk, S., Elmore, D., Card, G.L.: 2010, Polarization calibration of the Helioseismic and Magnetic Imager (HMI) onboard the Solar Dynamics Observatory (SDO). Solar Phys. doi:10.1007/s11207-010-9639-8.

Wachter, R., Schou, J.: 2009, Inferring small-scale flatfields from solar rotation. Solar Phys. 258, 331 - 341. doi:10.1007/s11207-009-9406-x.

Wachter, R., Schou, J., Rabello-Soares, M.C., Miles, J.W., Duvall, T.L., Bush, R.I.: 2011, Image quality of the Helioseismic and Magnetic Imager (HMI) onboard the Solar Dynamics Observatory (SDO). Solar Phys. doi:10.1007/s11207-011-9709-6. 